\title{
Small $x$ behavior of parton distributions. Analytical and "frozen" coupling constants. BFKL corrections
}

\section{Anatoly Kotikov*†}

BLThPh, Joint Institute for Nuclear Research, Dubna

E-mail: kotikovatheor.jinr.ru

It is shown that in the leading twist approximation of the Wilson operator product expansion with "frozen" and analytic strong coupling constants, Bessel-inspired behavior of the structure functions $F_{2}$ and $F_{2}^{c c}$ and also the derivative $\partial \ln F_{2} / \partial \ln (1 / x)$ at small $x$ values, obtained for a flat initial condition in the DGLAP evolution equations, leads to good agreement with the deep inelastic scattering experimental data from HERA.

XXI International Baldin Seminar on High Energy Physics Problems September 10-15, 2012

JINR, Dubna, Russia

* Speaker.

${ }^{\dagger}$ The work was supported in part by RFBR grant No. 10-02-01259-a. 


\section{Introduction}

The experimental data from HERA on the deep-inelastic scattering (DIS) structure function (SF) $F_{2}$ [1]-[3], its derivative $\partial \ln F_{2} / \partial \ln (1 / x)$ [4]-[6] and the heavy quark parts $F_{2}^{c c}$ and $F_{2}^{b b}$ [7][11] enable us to enter into a very interesting kinematical range for testing the theoretical ideas on the behavior of quarks and gluons carrying a very low fraction of momentum of the proton, the so-called small- $x$ region. In this limit one expects that the conventional treatment based on the Dokshitzer-Gribov-Lipatov-Altarelli-Parisi (DGLAP) equations [12] does not account for contributions to the cross section which are leading in $\alpha_{s} \ln (1 / x)$ and, moreover, the parton distribution function (PDFs), in particular the gluon ones, are becoming large and need to develop a high density formulation of QCD.

However, the reasonable agreement between HERA data and the next-to-leading-order (NLO) approximation of perturbative QCD has been observed for $Q^{2} \geq 2 \mathrm{GeV}^{2}$ (see reviews in [13] and references therein) and, thus, perturbative QCD could describe the evolution of $F_{2}$ and its derivatives up to very low $Q^{2}$ values, traditionally explained by soft processes.

The standard program to study the $x$ behavior of quarks and gluons is carried out by comparison of data with the numerical solution of the DGLAP equation [12] ${ }^{1}$ by fitting the parameters of the PDF $x$-profile at some initial $Q_{0}^{2}$ and the QCD energy scale $\Lambda$ [?]-[18]. However, for analyzing exclusively the low- $x$ region, there is the alternative of doing a simpler analysis by using some of the existing analytical solutions of DGLAP evolution in the low- $x$ limit [19]-[22]. This was done so in [19] where it was pointed out that the HERA small- $x$ data can be interpreted in terms of the so-called doubled asymptotic scaling (DAS) phenomenon related to the asymptotic behavior of the DGLAP evolution discovered many years ago [23].

The study of [19] was extended in [20,21,22] to include the finite parts of anomalous dimensions of Wilson operators ${ }^{2}$. This has led to predictions [21, 22] of the small- $x$ asymptotic PDF form in the framework of the DGLAP dynamics starting at some $Q_{0}^{2}$ with the flat function

$$
f_{a}\left(Q_{0}^{2}\right)=A_{a} \quad(\text { hereafter } a=q, g)
$$

where $f_{a}$ are the parton distributions multiplied by $x$ and $A_{a}$ are unknown parameters to be determined from the data.

We refer to the approach of $[20,21,22]$ as generalized DAS approximation. In that approach the flat initial conditions in Eq. (1.1) determine the basic role of the singular parts of anomalous dimensions, as in the standard DAS case, while the contribution from finite parts of anomalous dimensions and from Wilson coefficients can be considered as corrections which are, however, important for better agreement with experimental data. In the present paper, similary to [19]-[22], we neglect the contribution from the non-singlet quark component.

The use of the flat initial condition given in Eq. (1.1) is supported by the actual experimental situation: low- $Q^{2}$ data $[24,1,25,4]$ are well described for $Q^{2} \leq 0.4 \mathrm{GeV}^{2}$ by Regge theory with Pomeron intercept $\alpha_{P}(0) \equiv \lambda_{P}+1=1.08$, closed to the standard $\left(\alpha_{P}(0)=1\right)$ one. The small rise

\footnotetext{
${ }^{1}$ At small $x$ there is another approach based on the Balitsky-Fadin-Kuraev-Lipatov (BFKL) equation [14], whose application will be dicussed below in Appendix A.

${ }^{2}$ In the standard DAS approximation [23] only the singular parts of the anomalous dimensions were used.
} 
of HERA data $[1,4,25,26]$ at low $Q^{2}$ can be explained, for example, by contributions of higher twist operators (see [22]).

The purpose of this paper is to demostrate a good agreement between the predictions from the generalized DAS approach and the HERA experimental data [1, 2] and [7]-[11] for SF $F_{2}$ and $F_{2}^{c c}$ and also to compare the predictions for the slope $\partial \ln F_{2} / \partial \ln (1 / x)$ with the H1 and ZEUS data [5, 4, 6] (see Figs. 1-8). Looking at the H1 data points [1] shown in Figs. 5, 6 and 7 one can conclude that $\lambda\left(Q^{2}\right)$ is independent of $x$ within the experimental uncertainties for fixed $Q^{2}$ in the range $x<0.01$. Indeed, the data are well described by the power behavior

$$
F_{2}\left(x, Q^{2}\right)=C x^{-\lambda\left(Q^{2}\right)}
$$

where $\lambda\left(Q^{2}\right)=\hat{a} \ln \left(Q^{2} / \Lambda^{2}\right)$ with $C \approx 0.18, \hat{a} \approx 0.048$ and $\Lambda=292 \mathrm{MeV}$ [5]. The linear rise of the exponent $\lambda\left(Q^{2}\right)$ with $\ln Q^{2}$ is also explicitly shown in Figs. 5, 6 and 7 by the dashed line.

The rise of $\lambda\left(Q^{2}\right)$ linearly with $\ln Q^{2}$ can be tracted in strong nonperturbative way (see [27] and references therein), i.e., $\lambda\left(Q^{2}\right) \sim 1 / \alpha_{s}\left(Q^{2}\right)$. The previous analysis [28], however, demonstrated that the rise can be explained naturally in the framework of perturbative QCD.

The ZEUS and H1 Collaborations have also presented $[4,6]$ new preliminary data for $\lambda\left(Q^{2}\right)$ at quite low values of $Q^{2}$. As it is possible to see in Fig. 8 of [4], the ZEUS value for $\lambda\left(Q^{2}\right)$ is consistent with a constant $\sim 0.1$ at $Q^{2}<0.6 \mathrm{GeV}^{2}$, as it is expected under the assumption of single soft Pomeron exchange within the framework of Regge phenomenology. These points lie slightly below the corresponding ZEUS data but all the results are in agreement within modern experimental errors.

It is important to extend the analysis of $[21,22,28]$ to low $Q^{2}$ range with the help of wellknown infrared modifications of the strong coupling constant. Indeed, in Ref. [29], we have used the "frozen" and analytic versions (see, [35] and [36], respectively).

This contribution is organized as follows. Sections 2 and 3 contain basic formulae, which are needed for the present study and were previously obtained in [21, 22, 28, 29, 30]. In Sections 4 and 5 we compare our calculations with $\mathrm{H} 1$ and ZEUS experimental data and present the obtained results. Some discussions can be found in the conclusions. Some preliminary results accounting for BFKL corrections in our analysis can be found in Appendix A. It is hoped that the inclusion of these corrections will improve the agreement with the experimantal data for $F_{2}$ and its slope at $Q^{2} \sim 1 \div 2 \mathrm{Gev}^{2}$.

\section{Generalized DAS approach}

The flat initial condition (1.1) corresponds to the case when parton density tend to some constant value at $x \rightarrow 0$ and at some initial value $Q_{0}^{2}$. The main ingredients of the results [21,22], are:

- Both, the gluon and quark singlet densities are presented in terms of two components ("+" and "- ") which are obtained from the analytic $Q^{2}$-dependent expressions of the corresponding ("+" and "- ") PDF moments. ${ }^{3}$

\footnotetext{
${ }^{3}$ Such an approach has been developed [31] recently also for the fragmentation function, whose first moments (ie
} 
- The twist-two part of the" - " component is constant at small $x$ at any values of $Q^{2}$, whereas the one of the "+" component grows at $Q^{2} \geq Q_{0}^{2}$ as

$$
\sim e^{\sigma}, \quad \sigma=2 \sqrt{\left[\left|\hat{d}_{+}\right| s-\left(\hat{d}_{++}+\left|\hat{d}_{+}\right| \frac{\beta_{1}}{\beta_{0}}\right) p\right] \ln \left(\frac{1}{x}\right)}, \quad \rho=\frac{\sigma}{2 \ln (1 / x)},
$$

where $\sigma$ and $\rho$ are the generalized Ball-Forte variables,

$$
s=\ln \left(\frac{a_{s}\left(Q_{0}^{2}\right)}{a_{s}\left(Q^{2}\right)}\right), p=a_{s}\left(Q_{0}^{2}\right)-a_{s}\left(Q^{2}\right), \quad \hat{d}_{+}=-\frac{12}{\beta_{0}}, \quad \hat{d}_{++}=\frac{412}{27 \beta_{0}} .
$$

Hereafter we use the notation $a_{s}=\alpha_{s} /(4 \pi)$. The first two coefficients of the QCD $\beta$-function in the $\overline{\mathrm{MS}}$-scheme are $\beta_{0}=11-(2 / 3) f$ and $\beta_{1}=102-(114 / 9) f$ with $f$ is being the number of active quark flavors.

Note here that the perturbative coupling constant $a_{s}\left(Q^{2}\right)$ is different at the leading-order (LO) and NLO approximations. Indeed, from the renormalization group equation we can obtain the following equations for the coupling constant

$$
\frac{1}{a_{s}^{\mathrm{LO}}\left(Q^{2}\right)}=\beta_{0} \ln \left(\frac{Q^{2}}{\Lambda_{\mathrm{LO}}^{2}}\right)
$$

at the LO approximation and

$$
\frac{1}{a_{s}\left(Q^{2}\right)}+\frac{\beta_{1}}{\beta_{0}} \ln \left[\frac{\beta_{0}^{2} a_{s}\left(Q^{2}\right)}{\beta_{0}+\beta_{1} a_{s}\left(Q^{2}\right)}\right]=\beta_{0} \ln \left(\frac{Q^{2}}{\Lambda^{2}}\right)
$$

at the NLO approximation. Usually at the NLO level $\overline{\mathrm{MS}}$-scheme is used, so we apply $\Lambda=\Lambda_{\overline{\mathrm{MS}}}$ below.

\subsection{Parton distributions and the structure function $F_{2}$}

The results for parton densities and $F_{2}$ are following:

- The structure function $F_{2}$ has the form:

$$
\begin{aligned}
& F_{2, \mathrm{LO}}\left(x, Q^{2}\right)=e f_{q, \mathrm{LO}}\left(x, Q^{2}\right), \\
& f_{a, \mathrm{LO}}\left(x, Q^{2}\right)=f_{a, \mathrm{LO}}^{+}\left(x, Q^{2}\right)+f_{a, \mathrm{LO}}^{-}\left(x, Q^{2}\right)
\end{aligned}
$$

at the LO approximation, where

$$
e=\left(\sum_{1}^{f} e_{i}^{2}\right) / f
$$

is the average charge square, and

$$
\begin{aligned}
& F_{2}\left(x, Q^{2}\right)=e\left(f_{q}\left(x, Q^{2}\right)+\frac{2}{3} f a_{s}\left(Q^{2}\right) f_{g}\left(x, Q^{2}\right)\right), \\
& f_{a}\left(x, Q^{2}\right)=f_{a}^{+}\left(x, Q^{2}\right)+f_{a}^{-}\left(x, Q^{2}\right)
\end{aligned}
$$

at the NLO approximation.

mean multiplicities of quarks and gluons) were analyzed [32]. The results are in good agreement with the experimental data (see contribution [33] by Paolo Bolzoni to this Proceedings). 
- The small- $x$ asymptotic results for the LO parton densities $f_{a, \mathrm{LO}}^{ \pm}$are

$$
\begin{aligned}
f_{g, \mathrm{LO}}^{+}\left(x, Q^{2}\right) & =\left(A_{g}+\frac{4}{9} A_{q}\right) \tilde{I}_{0}\left(\sigma_{\mathrm{LO}}\right) e^{-\bar{d}_{+} s_{\mathrm{LO}}}+O\left(\rho_{\mathrm{LO}}\right) \\
f_{q, \mathrm{LO}}^{+}\left(x, Q^{2}\right) & =\frac{f}{9}\left(A_{g}+\frac{4}{9} A_{q}\right) \rho_{\mathrm{LO}} \tilde{I}_{1}\left(\sigma_{\mathrm{LO}}\right) e^{-\bar{d}_{+} s_{\mathrm{LO}}}+O(\rho) \\
f_{g, \mathrm{LO}}^{-}\left(x, Q^{2}\right) & =-\frac{4}{9} A_{q} e^{-d_{-} s_{\mathrm{LO}}}+O(x) \\
f_{q, \mathrm{LO}}^{-}\left(x, Q^{2}\right) & =A_{q} e^{-d_{-} s_{\mathrm{LO}}}+O(x)
\end{aligned}
$$

where

$$
\bar{d}_{+}=1+20 f /\left(27 \beta_{0}\right), d_{-}=16 f /\left(27 \beta_{0}\right)
$$

are the regular parts of the anomalous dimensions $d_{+}(n)$ and $d_{-}(n)$, respectively, in the limit $n \rightarrow 1^{4}$. Here $n$ is the variable in Mellin space. The functions $\tilde{I}_{v}(v=0,1)$ are related to the modified Bessel function $I_{v}$ and to the Bessel function $J_{v}$ by:

$$
\tilde{I}_{v}(\sigma)=\left\{\begin{array}{ll}
I_{v}(\sigma), & \text { if } s \geq 0 \\
i^{-v} J_{v}(i \sigma), i^{2}=-1, & \text { if } s \leq 0
\end{array} .\right.
$$

At the LO, the variables $\sigma_{\mathrm{LO}}$ and $\rho_{\mathrm{LO}}$ are given by Eq. (2.1) when $p=0$, i.e.

$$
\sigma_{\mathrm{LO}}=2 \sqrt{\left|\hat{d}_{+}\right| s_{\mathrm{LO}} \ln \left(\frac{1}{x}\right)}, \quad \rho_{\mathrm{LO}}=\frac{\sigma_{\mathrm{LO}}}{2 \ln (1 / x)},
$$

and the variable $s_{\mathrm{LO}}$ is given by Eq. (2.2) with $a_{s}^{\mathrm{LO}}\left(Q^{2}\right)$ as in Eq. (2.3).

- The small- $x$ asymptotic results for the NLO parton densities $f_{a}^{ \pm}$are

$$
\begin{aligned}
f_{g}^{+}\left(x, Q^{2}\right) & =A_{g}^{+}\left(Q^{2}, Q_{0}^{2}\right) \tilde{I}_{0}(\sigma) e^{-\bar{d}_{+} s-\bar{D}_{+} p}+O(\rho) \\
f_{q}^{+}\left(x, Q^{2}\right) & =A_{q}^{+}\left[\left(1-\bar{d}_{+-}^{q} a_{s}\left(Q^{2}\right)\right) \rho \tilde{I}_{1}(\sigma)+20 a_{s}\left(Q^{2}\right) I_{0}(\sigma)\right] e^{-\bar{d}_{+}(1) s-\bar{D}_{+} p}+O(\rho), \\
f_{g}^{-}\left(x, Q^{2}\right) & =A_{g}^{-}\left(Q^{2}, Q_{0}^{2}\right) e^{-d_{-}(1) s-D_{-} p}+O(x) \\
f_{q}^{-}\left(x, Q^{2}\right) & =A_{q}^{-} e^{-d_{-}(1) s-D_{-} p}+O(x)
\end{aligned}
$$

where

$$
D_{ \pm}=d_{ \pm \pm}-\frac{\beta_{1}}{\beta_{0}} d_{ \pm}
$$

and similar for $\hat{D}_{+}$and $\bar{D}_{+}$,

$$
\begin{aligned}
& A_{g}^{+}\left(Q^{2}, Q_{0}^{2}\right)=\left(1-\frac{80 f}{81} a_{s}(Q)\right) A_{g}+\frac{4}{9}\left(1+\left(3+\frac{f}{27}\right) a_{s}\left(Q_{0}\right)-\frac{80 f}{81} a_{s}(Q)\right) A_{q} \\
& A_{g}^{-}\left(Q^{2}, Q_{0}^{2}\right)=A_{g}-A_{g}^{+}\left(Q_{0}^{2}, Q^{2}\right) .
\end{aligned}
$$

\footnotetext{
${ }^{4}$ We denote the singular and regular parts of a given quantity $k(n)$ in the limit $n \rightarrow 1$ by $\hat{k} /(n-1)$ and $\bar{k}$, respectively.
} 
The coupling constant $a_{s}\left(Q^{2}\right)$ is introduced in Eq. (2.4). The variables $\hat{d}_{+}, \hat{d}_{++} \bar{d}_{+}$and $d_{-}$ are diven in Eqs. (2.2) and (2.12), respectively. The variables $\bar{d}_{++}, d_{--}$and $d_{+-}^{q}$ have the form

$$
\begin{aligned}
& \bar{d}_{++}=\frac{8}{\beta_{0}}\left(36 \zeta_{3}+33 \zeta_{2}-\frac{1643}{12}+\frac{2 f}{9}\left[\frac{68}{9}-4 \zeta_{2}-\frac{13 f}{243}\right]\right) \\
& d_{--}=\frac{16}{9 \beta_{0}}\left(2 \zeta_{3}-3 \zeta_{2}+\frac{13}{4}+f\left[4 \zeta_{2}-\frac{23}{18}+\frac{13 f}{243}\right]\right), d_{+-}^{q}=23-12 \zeta_{2}-\frac{13 f}{81}
\end{aligned}
$$

with $\zeta_{3}$ and $\zeta_{2}$ are Eller functions. ${ }^{5}$

\subsection{Effective slopes}

Contrary to the approach in [19]-[22] various groups have been able to fit the available data using a hard input at small $x: x^{-\lambda}, \lambda>0$ with different $\lambda$ values at low and high $Q^{2}$ (see [37]-[43]). Such results are well-known at low $Q^{2}$ values [38]. At large $Q^{2}$ values, for the modern HERA data it is also not very surprising, because they cannot distinguish between the behavior based on a steep input parton parameterization, at quite large $Q^{2}$, and the steep form acquired after the dynamical evolution from a flat initial condition at quite low $Q^{2}$ values.

As it has been mentioned above and shown in [21, 22], the behavior of parton densities and $F_{2}$ given in the Bessel-like form by generalized DAS approach can mimic a power law shape over a limited region of $x$ and $Q^{2}$

$$
f_{a}\left(x, Q^{2}\right) \sim x^{-\lambda_{a}^{\text {eff }}\left(x, Q^{2}\right)} \text { and } F_{2}\left(x, Q^{2}\right) \sim x^{-\lambda_{\mathrm{F}_{2}}^{\mathrm{eff}}\left(x, Q^{2}\right)} .
$$

The effective slopes $\lambda_{a}^{\text {eff }}\left(x, Q^{2}\right)$ and $\lambda_{\mathrm{F}_{2}}^{\text {eff }}\left(x, Q^{2}\right)$ have the form:

$$
\begin{aligned}
& \lambda_{g}^{\text {eff }}\left(x, Q^{2}\right)=\frac{f_{g}^{+}\left(x, Q^{2}\right)}{f_{g}\left(x, Q^{2}\right)} \rho \frac{\tilde{I}_{1}(\sigma)}{\tilde{I}_{0}(\sigma)} \\
& \lambda_{q}^{\text {eff }}\left(x, Q^{2}\right)=\frac{f_{q}^{+}\left(x, Q^{2}\right)}{f_{q}\left(x, Q^{2}\right)} \rho \frac{\tilde{I}_{2}(\sigma)\left(1-20 a_{s}\left(Q^{2}\right)\right)+20 a_{s}\left(Q^{2}\right) \tilde{I}_{1}(\sigma) / \rho}{\tilde{I}_{1}(\sigma)\left(1-20 a_{s}\left(Q^{2}\right)\right)+20 a_{s}\left(Q^{2}\right) \tilde{I}_{0}(\sigma) / \rho} \\
& \lambda_{\mathrm{F}_{2}}^{\text {eff }}\left(x, Q^{2}\right)=\frac{\lambda_{q}^{e f f}\left(x, Q^{2}\right) f_{q}^{+}\left(x, Q^{2}\right)+(2 f) / 3 a_{s}\left(Q^{2}\right) \lambda_{g}^{e f f}\left(x, Q^{2}\right) f_{g}^{+}\left(x, Q^{2}\right)}{f_{q}\left(x, Q^{2}\right)+(2 f) / 3 a_{s}\left(Q^{2}\right) f_{g}\left(x, Q^{2}\right)}
\end{aligned}
$$

where the exact form of parton densities can be found in [21, 22].

The results (2.22) (and also (2.23)-(2.25) below) are given at the NLO approximation. To obtain the LO one, it is necessary to cancel the term $\sim a_{s}\left(Q^{2}\right)$ and to use Eqs. (2.8)-(2.11) for parton densities $f_{a}\left(x, Q^{2}\right)$.

The effective slopes $\lambda_{a}^{\text {eff }}$ and $\lambda_{\mathrm{F}_{2}}^{\text {eff }}$ depend on the magnitudes $A_{a}$ of the initial PDFs and also on the chosen input values of $Q_{0}^{2}$ and $\Lambda$. To compare with the experimental data it is necessary the exact expressions (2.22), but for qualitative analysis it is better to use an approximation.

\footnotetext{
${ }^{5}$ Note that evaluation of the results (2.8)-(2.21) need the knowledge of the analytic continuation of the anomalous dimansions and coefficient functions. The analytic continuation can be found in Refs. [34]. It was used also for the fits [17, 18].
} 


\subsection{Asymptotic form of the effective slopes}

At quite large values of $Q^{2}$, where the "-" component is negligible, the dependence on the initial PD disappears, having in this case for the asymptotic behavior the following expressions ${ }^{6}$ :

$$
\begin{aligned}
\lambda_{g}^{\text {eff,as }}\left(x, Q^{2}\right) & =\rho \frac{\tilde{I}_{1}(\sigma)}{\tilde{I}_{0}(\sigma)} \approx \rho-\frac{1}{4 \ln (1 / x)}, \\
\lambda_{q}^{\text {eff,as }}\left(x, Q^{2}\right) & =\rho \frac{\tilde{I}_{2}(\sigma)\left(1-20 a_{s}\left(Q^{2}\right)+20 a_{s}\left(Q^{2}\right) \tilde{I}_{1}(\sigma) / \rho\right.}{\tilde{I}_{1}(\sigma)\left(1-20 a_{s}\left(Q^{2}\right)+20 a_{s}\left(Q^{2}\right) \tilde{I}_{0}(\sigma) / \rho\right.} \\
& \approx \rho-\frac{3}{4 \ln (1 / x)}+\frac{10 a_{s}\left(Q^{2}\right)}{\rho \ln (1 / x)}, \\
\lambda_{\mathrm{F}_{2}}^{\text {eff,as }}\left(x, Q^{2}\right) & =\rho \frac{\tilde{I}_{2}(\sigma)}{\tilde{I}_{1}(\sigma)}+26 a_{s}\left(Q^{2}\right)\left(1-\frac{\tilde{I}_{0}(\sigma) \tilde{I}_{2}(\sigma)}{\tilde{I}_{1}^{2}(\sigma)}\right) \\
& \approx \rho-\frac{3}{4 \ln (1 / x)}+\frac{13 a_{s}\left(Q^{2}\right)}{\rho \ln (1 / x)}=\lambda_{q}^{\text {eff,as }}\left(x, Q^{2}\right)+\frac{3 a_{s}\left(Q^{2}\right)}{\rho \ln (1 / x)},
\end{aligned}
$$

where the symbol $\approx$ marks the approximation obtained in the expansion of the usual and modified Bessel functions in (2.13). These approximations are accurate only at very large $\sigma$ values (i.e. at very large $Q^{2}$ and/or very small $x$ ).

As one can see from Eqs. (2.23) and (2.24), the gluon effective slope $\lambda_{g}^{\text {eff }}$ is larger than the quark slope $\lambda_{q}^{\text {eff }}$, which is in excellent agreement with MRS [44] and GRV [45] analyses.

We would like to note that at the NLO approximation the slope $\lambda_{\mathrm{F}_{2}}^{\text {eff,as }}\left(x, Q^{2}\right)$ lies between quark and gluon ones but closely to quark slope $\lambda_{q}^{\text {eff,as }}\left(x, Q^{2}\right)$. Indeed,

$$
\begin{aligned}
\lambda_{g}^{\text {eff,as }}\left(x, Q^{2}\right)-\lambda_{\mathrm{F}_{2}}^{\text {eff,as }}\left(x, Q^{2}\right) & \approx\left(\rho-\frac{1}{4 \ln (1 / x)}+26 a_{s}\left(Q^{2}\right)\right) \frac{1}{2 \rho \ln (1 / x)}, \\
\lambda_{\mathrm{F}_{2}}^{\text {eff,as }}\left(x, Q^{2}\right)-\lambda_{q}^{\text {eff,as }}\left(x, Q^{2}\right) & \approx \frac{3 a_{s}\left(Q^{2}\right)}{\rho \ln (1 / x)} .
\end{aligned}
$$

Both slopes $\lambda_{a}^{\text {eff }}\left(x, Q^{2}\right)$ decrease with increasing $x$ (see Fig. 5). A $x$-dependence of the slope should not appear for PDFs within a Regge type asymptotic $\left(x^{-\lambda}\right)$ and precise measurement of the slope $\lambda_{a}^{\text {eff }}\left(x, Q^{2}\right)$ may lead to the possibility to verify the type of small- $x$ asymptotics of parton distributions.

\section{3. $F_{2}^{c c}$ and $F_{2}^{b b}$ structure functions}

Recently the H1 $[7,8]$ and ZEUS $[9,10]$ Collaborations at HERA presented new data ${ }^{7}$. Moreover, the preliminary combine H1 and ZEUS data of $F_{2}^{c c}\left(x, Q^{2}\right)$ and $F_{2}^{b b}\left(x, Q^{2}\right)$ has been demonstrated recently (see [11]).

\footnotetext{
${ }^{6}$ The asymptotic formulae given in Eqs. (2.23)-(2.25) work quite well at any $Q^{2} \geq Q_{0}^{2}$ values, because at $Q^{2}=Q_{0}^{2}$ the values of $\lambda_{a}^{\text {eff }}$ and $\lambda_{\mathrm{F}_{2}}^{\text {eff }}$ are equal zero. The use of approximations in Eqs. (2.23)-(2.25) instead of the exact results given in Eq. (2.22) underestimates (overestimates) only slightly the gluon (quark) slope at $Q^{2} \geq Q_{0}^{2}$.

${ }^{7}$ The papers [7]-[10] contain also the references on the previous data on deep-inelastic (DIS) structure functions (SFs) $F_{2}^{c c}$ and $F_{2}^{b b}$ at small $x$ values.
} 
In the framework of DGLAP dynamics [12], there are two basic methods to study heavyflavour physics. One of them [46] is based on the massless PDF evolution of parton and the other one on the photon-gluon fusion (PGF) process [47]. There are also some interpolating schemes (see Ref. [48] and references cited therein).

Here we present the results of Ref. [49] were we applied compact low- $x$ approximation formulae for the SFs $F_{2}^{i i}\left(x, Q^{2}\right)$, with hereafter $i=c, b$, observed [30] in the framework of PGF process at the first two orders of perturbation theory to these new HERA experimental data [7]-[11]. We show a good agreement between experimental data and the approach which found without additional free parameters. All PDF parameters have been fitted earlier [22, 29] from $F_{2}\left(x, Q^{2}\right)$ HERA experimental data.

In the framework of the generalized DAS approach, the SFs $F_{2}^{c c}\left(x, Q^{2}\right)$ and $F_{2}^{b b}\left(x, Q^{2}\right)$ have the following form

$$
F_{2}^{i i}\left(x, Q^{2}\right) \approx M_{2, g}^{i}\left(1, Q^{2}, \mu^{2}\right) f_{g}\left(x, \mu^{2}\right), \quad(i=c, b)
$$

where $M_{2, g}^{i}\left(1, Q^{2}, \mu^{2}\right)$ is the first Mellin moment of the so-called gluon coefficient function $C_{2, g}^{i}\left(x, Q^{2}, \mu^{2}\right)$.

Through NLO, $M_{2, g}\left(1, Q^{2}, \mu^{2}\right)$ exhibits the structure

$$
M_{2, g}^{i}\left(1, Q^{2}, \mu^{2}\right)=e_{i}^{2} a_{s}(\mu)\left\{M_{2, g}^{(0)}\left(1, c_{i}\right)+a_{s}(\mu)\left[M_{2, g}^{(1)}\left(1, c_{i}\right)+M_{2, g}^{(2)}\left(1, c_{i}\right) \ln \frac{\mu^{2}}{m_{i}^{2}}\right]\right\}+\mathscr{O}\left(a_{s}^{3}\right),
$$

where

$$
c_{i}=\frac{m_{i}^{2}}{Q^{2}}, \quad \mu^{2}=Q^{2}+4 m_{i}^{2}
$$

\subsection{LO results}

The LO coefficient function of PGF can be obtained from the QED case [59] by adjusting coupling constants and colour factors, and they read $[60,61]$

$$
C_{2, g}^{(0)}(x, c)=-2 x\left\{[1-4 x(2-c)(1-x)] \beta-\left[1-2 x(1-2 c)+2 x^{2}\left(1-6 c-4 c^{2}\right)\right] L(\beta)\right\},
$$

where

$$
\beta(x)=\sqrt{1-\frac{4 c x}{1-x}}, \quad L(\beta)=\ln \frac{1+\beta}{1-\beta} .
$$

Performing the Mellin transformation

$$
M_{2, g}(n, c)=\int_{0}^{b} \frac{d x}{x} C_{2, g}(x, c)
$$

we find at $n=1$ (see [30]) ${ }^{8}$

$$
M_{2, g}^{(0)}(1, c)=\frac{2}{3}[1+2(1-c) J(c)]
$$

with

$$
J(c)=-\sqrt{b} \ln t, \quad t=\frac{1-\sqrt{b}}{1+\sqrt{b}}, \quad b=\frac{1}{1+4 c} .
$$

\footnotetext{
${ }^{8}$ Note that similar formulas work well for (see [62]) for high-energy neutrino-nucleo scattering where the effective value of the Bjorken variable $x$ is very small.
} 


\subsection{NLO results}

The NLO coefficient functions of PGF are rather lengthy and not published in print; they are only available as computer codes [63]. For the purpose of this letter, it is sufficient to work in the high-energy regime, defined by $x \ll 1$, where they assume the compact form [64]

$$
C_{2, g}^{(j)}(x, c)=\beta R_{2, g}^{(j)}(1, c),
$$

with

$$
R_{2, g}^{(1)}(1, c)=\frac{8}{9} C_{A}[5+(13-10 c) J(c)+6(1-c) I(c)], \quad R_{2, g}^{(2)}(1, c)=-4 C_{A} M_{2, g}^{(0)}(1, c),
$$

where $C_{A}=N$ for the colour gauge group $\mathrm{SU}(\mathrm{N}), J(c)$ is defined by Eq. (3.8), and

$$
I(c)=-\sqrt{b}\left[\zeta(2)+\frac{1}{2} \ln ^{2} t-\ln (b c) \ln t+2 \mathrm{Li}_{2}(-t)\right],
$$

where $t$ is given in (3.8) and $\operatorname{Li}_{2}(x)=-\int_{0}^{1}(d y / y) \ln (1-x y)$ is the dilogarithmic function.

As already mentioned above (see the end of Section 2), the Mellin transforms of $C_{k, g}^{(j)}(x, c)$ exhibit singularities in the limit $\delta_{ \pm} \rightarrow 0$, which lead to modifications in Eq. (3.1). As was shown in Refs. [40,21,22], the terms involving $1 / \delta_{ \pm}$correspond to singularities of the Mellin moments $M_{2, g}^{ \pm}(n)$ at $n \rightarrow 1$ and depend on the exact form of the subasymptotic low- $x$ behaviour encoded in $\tilde{f}_{g}^{ \pm}\left(x, \mu^{2}\right)$. The modification is simple:

$$
\frac{1}{\delta_{ \pm}} \rightarrow \frac{1}{\tilde{\delta}_{ \pm}}, \quad \frac{1}{\tilde{\delta}_{ \pm}}=\frac{1}{\tilde{f}_{g}^{ \pm}\left(\hat{x}, \mu^{2}\right)} \int_{\hat{x}}^{1} \frac{d y}{y} \tilde{f}_{g}^{ \pm}\left(y, \mu^{2}\right)
$$

where $\hat{x}=x / b$. In the generalized DAS regime, the + and - components of the gluon PDF exhibit the low- $x$ behaviour (2.15)-(2.21). We thus have [21, 22]

$$
\frac{1}{\tilde{\delta}_{+}} \approx \frac{1}{\rho(\hat{x})} \frac{I_{1}(\sigma(\hat{x}))}{I_{0}(\sigma(\hat{x}))}, \quad \frac{1}{\tilde{\delta}_{-}} \approx \ln \frac{1}{\hat{x}}
$$

where $\sigma$ and $\rho$ are given in (2.1).

Because the ratio $f_{g}^{-}\left(x, Q^{2}\right) / f_{g}^{+}\left(x, Q^{2}\right)$ is rather small at the $Q^{2}$ values considered, Eq. (3.1) is modified to become

$$
F_{2}^{i i}\left(x, Q^{2}\right) \approx \tilde{M}_{2, g}\left(1, \mu^{2}, c_{i}\right) f_{g}\left(x, \mu^{2}\right),
$$

where $\tilde{M}_{2, g}\left(1, \mu^{2}\right)$ is obtained from $M_{2, g}\left(n, \mu^{2}\right)$ by taking the limit $n \rightarrow 1$ and replacing $1 /(n-1) \rightarrow$ $1 / \tilde{\delta}_{+}$. Consequently, one needs to substitute

$$
M_{2, g}^{(j)}(1, c) \rightarrow \tilde{M}_{2, g}^{(j)}(1, c) \quad(j=1,2)
$$

in the NLO part of Eq. (3.2). Using the identity

$$
\frac{1}{I_{0}(\sigma(\hat{x}))} \int_{\hat{x}}^{1} \frac{d y}{y} \beta\left(\frac{x}{y}\right) I_{0}(\sigma(y)) \approx \frac{1}{\tilde{\delta}_{+}}-\ln (b c)-\frac{J(c)}{b},
$$




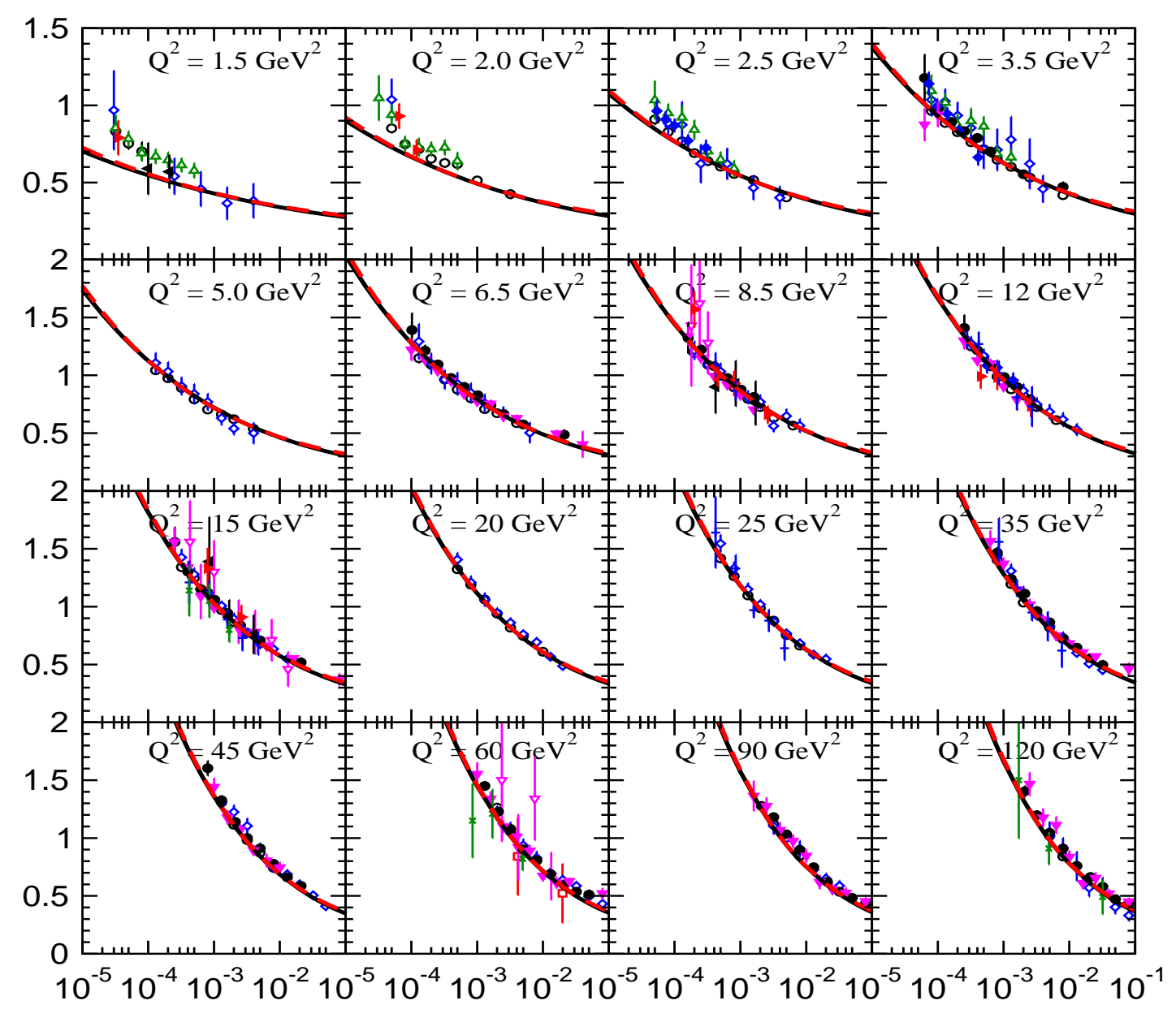

Figure 1: $F_{2}\left(x, Q^{2}\right)$ as a function of $x$ for different $Q^{2}$ bins. The experimental points are from H1 [1] (open points) and ZEUS [2] (solid points) at $Q^{2} \geq 1.5 \mathrm{GeV}^{2}$. The solid curve represents the NLO fit. The dashed curve (hardly distinguishable from the solid one) represents the LO fit.

we find the Mellin transform of Eq. (3.9) to be ${ }^{9}$

$$
\tilde{M}_{2, g}^{(j)}(1, c) \approx\left[\frac{1}{\delta_{+}}-\ln (b c)-\frac{J(c)}{b}\right] R_{2, g}^{(j)}(1, c) \quad(j=1,2),
$$

with $R_{2, g}^{(j)}(1, a) \quad(j=1,2)$ are given in (3.10). The rise of the NLO terms as $x \rightarrow 0$ is in agreement with earlier investigations [65].

\section{Comparison with experimental data for SF $F_{2}$ and the slope $\lambda_{\mathrm{F}_{2}}$}

\footnotetext{
${ }^{9}$ Note, that $\delta_{+}$determines the behavior of the slope of gluon density (see (2.22)) and also mostly the slope of SF $F_{2}$. The form (3.13) of $\tilde{\delta}_{+}$is in full agreement with the results (2.22) for the asymptotic form of the effective slope of gluon density.
} 


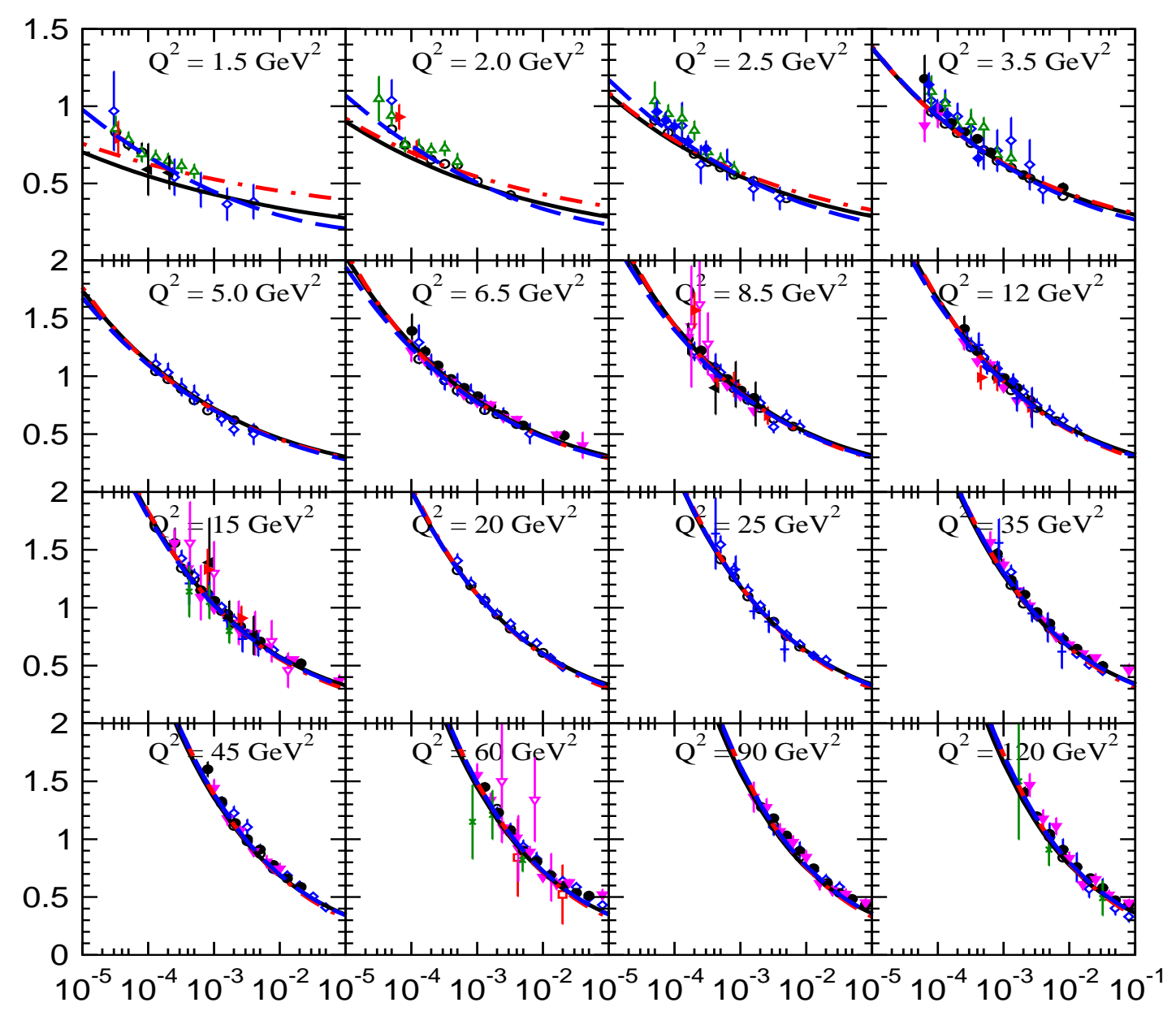

Figure 2: $F_{2}\left(x, Q^{2}\right)$ as a function of $x$ for different $Q^{2}$ bins. The experimental points are same as on Fig. 1. The solid curve represents the NLO fit. The dash-dotted curve represents the BFKL-motivated estimation for higher-twist corrections to $F_{2}\left(x, Q^{2}\right)$ (see [22]). The dashed curve is obtained from the fits at the NLO, when the renormalon contributions of higher-twist terms have been incorporated.

Using the results of previous section we have analyzed HERA data for $F_{2}$ and the slope $\partial \ln F_{2} / \partial \ln (1 / x)$ at small $x$ from the H1 and ZEUS Collaborations [1]-[6].

In order to keep the analysis as simple as possible, we fix $f=4$ and $\alpha_{s}\left(M_{Z}^{2}\right)=0.1166$ (i.e., $\Lambda^{(4)}=284 \mathrm{MeV}$ ) in agreement with the more recent ZEUS results [2].

As it is possible to see in Fig. 1 (see also [21, 22]), the twist-two approximation is reasonable at $Q^{2} \geq 2 \mathrm{GeV}^{2}$. At smaller $Q^{2}$, some modification of the approximation should be considered. In Ref. [22] we have added the higher twist corrections. For renormalon model of higher twists, we have found a good agreement with experimental data at essentially lower $Q^{2}$ values: $Q^{2} \geq 0.5$ $\mathrm{GeV}^{2}$ (see Figs. 2 and 3), but we have added 4 additional parameters: amplitudes of twist- 4 and twist- 6 corrections to quark and gluon densities.

Moreover, the results of fits in [22] have an important property: they are very similar in LO and 


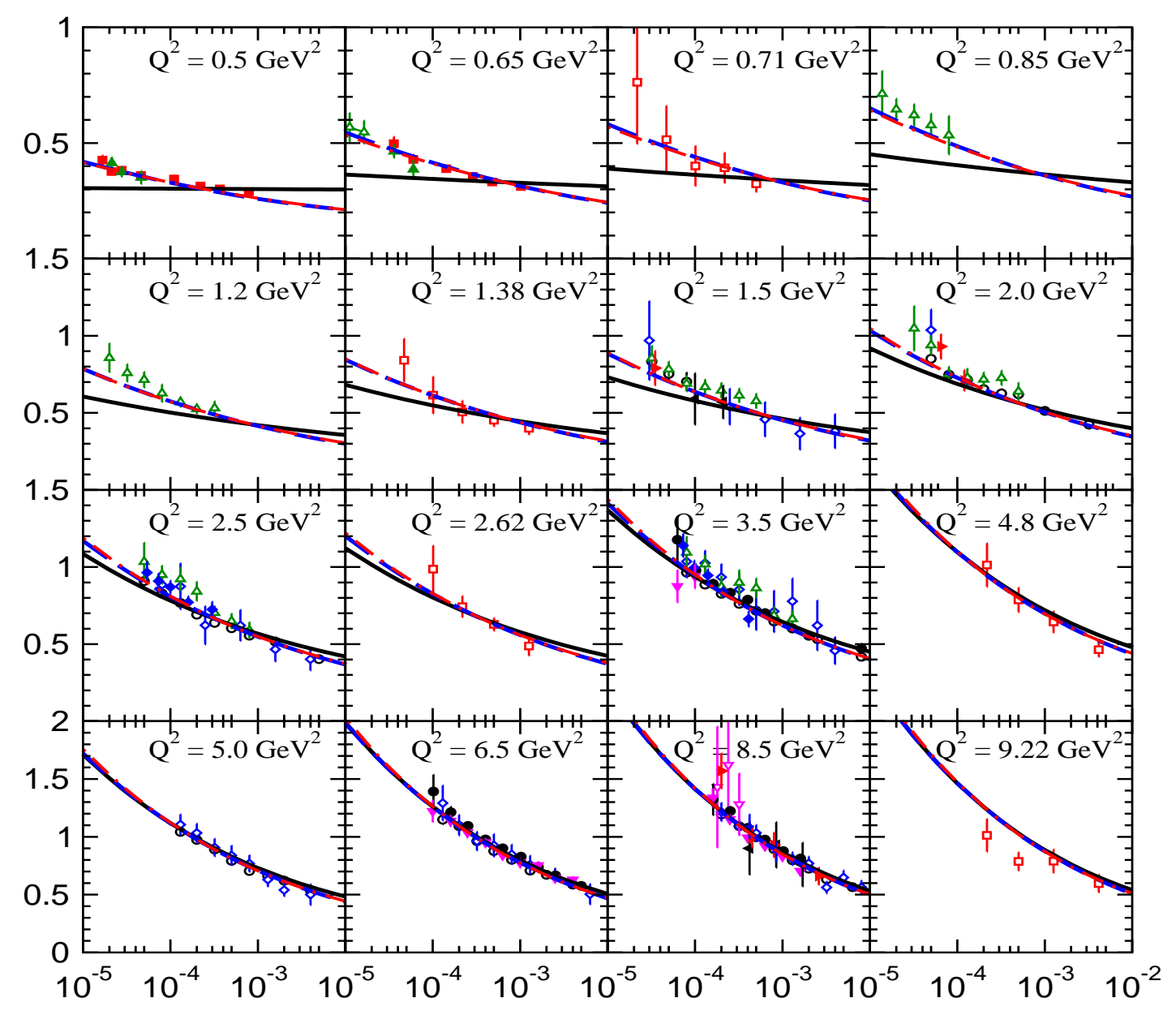

Figure 3: $F_{2}\left(x, Q^{2}\right)$ as a function of $x$ for different $Q^{2}$ bins. The experimental points are from H1 [1] (open points) and ZEUS [2] (solid points) at $Q^{2} \geq 0.5 \mathrm{GeV}^{2}$. The solid curve represents the NLO fit. The dashed curve is from the fits at the NLO with the renormalon contributions of higher-twist terms incorporated. The dash-dotted curve (hardly distinguishable from the dashed one) represents the LO fit with the renormalon contributions of higher-twist terms incorporated.

NLO approximations of perturbation theory. The similarity is related to the fact that the small- $x$ asymptotics of the NLO corrections are usually large and negative (see, for example, $\alpha_{s}$-corrections $[50,51]$ to BFKL kernel $\left.[14]^{10}\right)$. Then, the LO form $\sim \alpha_{s}\left(Q^{2}\right)$ for some observable and the NLO one $\sim \alpha_{s}\left(Q^{2}\right)\left(1-K \alpha_{s}\left(Q^{2}\right)\right)$ with a large value of $K$ are similar, because $\Lambda \gg \Lambda_{\mathrm{LO}}{ }^{11}$ and, thus, $\alpha_{s}\left(Q^{2}\right)$ at LO is considerably smaller then $\alpha_{s}\left(Q^{2}\right)$ at NLO for HERA $Q^{2}$ values.

In other words, performing some resummation procedure (such as Grunberg's effective-charge method [52]), one can see that the results up to NLO approximation may be represented as $\sim$

\footnotetext{
${ }^{10}$ It seems that it is a property of any processes in which gluons, but not quarks play a basic role.

${ }^{11}$ The equality of $\alpha_{s}\left(M_{Z}^{2}\right)$ at LO and NLO approximations, where $M_{Z}$ is the Z-boson mass, relates $\Lambda$ and $\Lambda_{\mathrm{LO}}$ : $\Lambda^{(4)}=284 \mathrm{MeV}$ (as in [2]) corresponds to $\Lambda_{\mathrm{LO}}=112 \mathrm{MeV}$ (see [22]).
} 


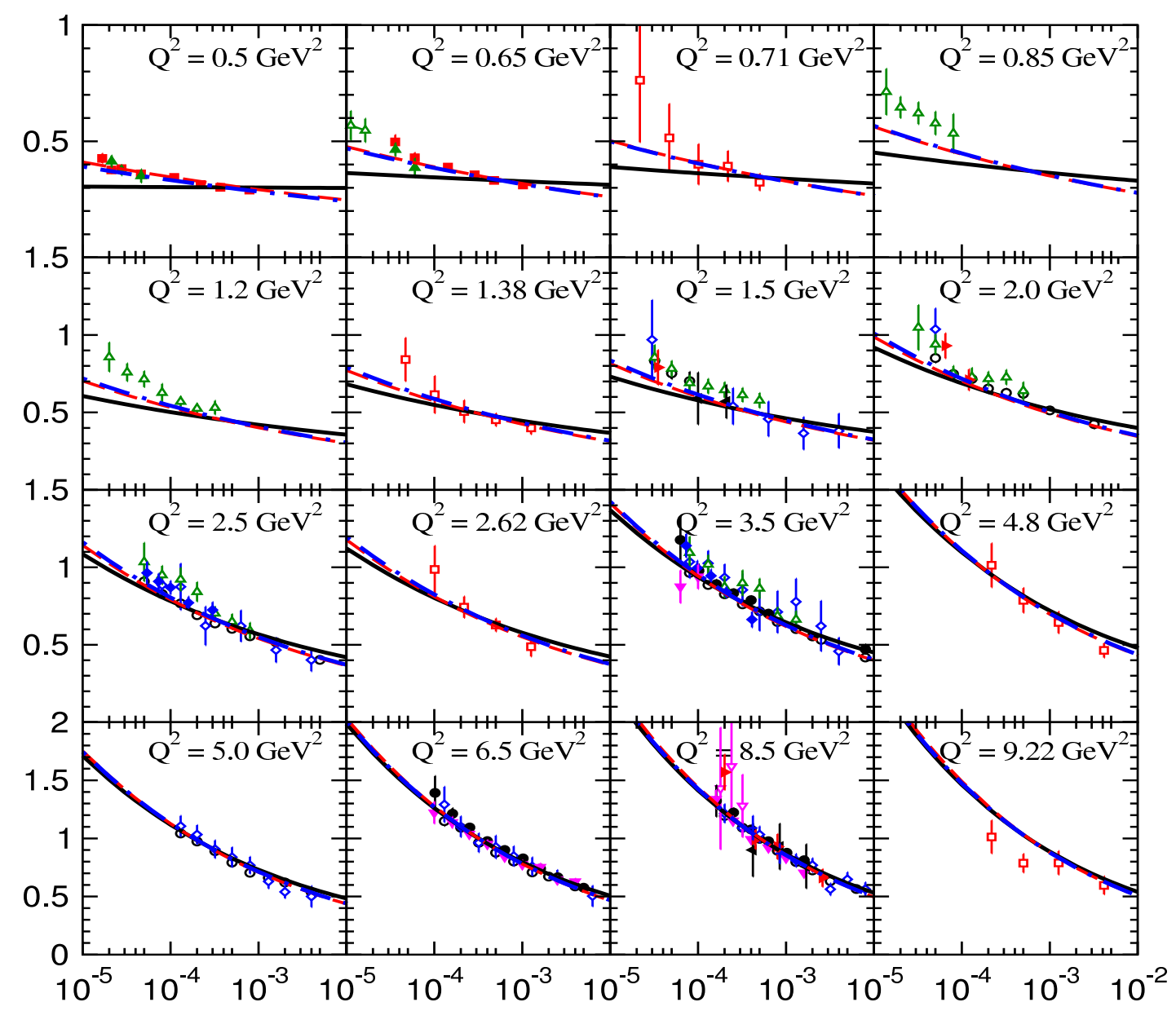

Figure 4: $x$ dependence of $F_{2}\left(x, Q^{2}\right)$ in bins of $Q^{2}$. The experimental data from $\mathrm{H} 1$ (open points) and ZEUS (solid points) are compared with the NLO fits for $Q^{2} \geq 0.5 \mathrm{GeV}^{2}$ implemented with the canonical (solid lines), frozen (dot-dashed lines), and analytic (dashed lines) versions of the strong-coupling constant. For comparison, also the results obtained in Ref. [22] through a fit based on the renormalon model of higher-twist terms are shown (dotted lines).

$\alpha_{s}\left(Q_{\text {eff }}^{2}\right)$, where $Q_{\text {eff }}^{2} \gg Q^{2}$. Indeed, from different studies [53, 54, 55], it is well known that at small- $x$ values the effective argument of the coupling constant is higher then $Q^{2}$.

Here, to improve the agreement at small $Q^{2}$ values without additional parameters, we modify the QCD coupling constant. We consider two modifications, which effectively increase the argument of the coupling constant at small $Q^{2}$ values (in agreement with $[53,54,55]$ ).

In one case, which is more phenomenological, we introduce freezing of the coupling constant by changing its argument $Q^{2} \rightarrow Q^{2}+M_{\rho}^{2}$, where $M_{\rho}$ is the $\rho$-meson mass (see [35]). Thus, in the formulae of the Section 2 we should do the following replacement:

$$
a_{s}\left(Q^{2}\right) \rightarrow a_{\mathrm{fr}}\left(Q^{2}\right) \equiv a_{s}\left(Q^{2}+M_{\rho}^{2}\right)
$$


Table 1: The result of the LO and NLO fits to H1 and ZEUS data for different low $Q^{2}$ cuts. In the fits $f$ is fixed to 4 flavors.

\begin{tabular}{|l||c|c|c||r|}
\hline \hline & $A_{g}$ & $A_{q}$ & $Q_{0}^{2}\left[\mathrm{GeV}^{2}\right]$ & $\chi^{2} /$ n.o.p. \\
\hline \hline$Q^{2} \geq 1.5 \mathrm{GeV}^{2}$ & & & & \\
LO & $0.784 \pm .016$ & $0.801 \pm .019$ & $0.304 \pm .003$ & $754 / 609$ \\
LO\&an. & $0.932 \pm .017$ & $0.707 \pm .020$ & $0.339 \pm .003$ & $632 / 609$ \\
LO\&fr. & $1.022 \pm .018$ & $0.650 \pm .020$ & $0.356 \pm .003$ & $547 / 609$ \\
\hline NLO & $-0.200 \pm .011$ & $0.903 \pm .021$ & $0.495 \pm .006$ & $798 / 609$ \\
NLO\&an. & $0.310 \pm .013$ & $0.640 \pm .022$ & $0.702 \pm .008$ & $655 / 609$ \\
NLO\&fr. & $0.180 \pm .012$ & $0.780 \pm .022$ & $0.661 \pm .007$ & $669 / 609$ \\
\hline \hline$Q^{2} \geq 0.5 \mathrm{GeV}^{2}$ & & & & \\
LO & $0.641 \pm .010$ & $0.937 \pm .012$ & $0.295 \pm .003$ & $1090 / 662$ \\
LO\&an. & $0.846 \pm .010$ & $0.771 \pm .013$ & $0.328 \pm .003$ & $803 / 662$ \\
LO\&fr. & $1.127 \pm .011$ & $0.534 \pm .015$ & $0.358 \pm .003$ & $679 / 662$ \\
\hline NLO & $-0.192 \pm .006$ & $1.087 \pm .012$ & $0.478 \pm .006$ & $1229 / 662$ \\
NLO\&an. & $0.281 \pm .008$ & $0.634 \pm .016$ & $0.680 \pm .007$ & $633 / 662$ \\
NLO\&fr. & $0.205 \pm .007$ & $0.650 \pm .016$ & $0.589 \pm .006$ & $670 / 662$ \\
\hline \hline
\end{tabular}

The second possibility incorporates the Shirkov-Solovtsov idea $[36,56,57]$ about analyticity of the coupling constant that leads to the additional its power dependence. Then, in the formulae of the previous section the coupling constant $a_{s}\left(Q^{2}\right)$ should be replaced as follows:

$$
a_{\mathrm{an}}^{\mathrm{LO}}\left(Q^{2}\right)=a_{s}\left(Q^{2}\right)-\frac{1}{\beta_{0}} \frac{\Lambda_{\mathrm{LO}}^{2}}{Q^{2}-\Lambda_{\mathrm{LO}}^{2}}
$$

at the LO approximation and

$$
a_{\mathrm{an}}\left(Q^{2}\right)=a_{s}\left(Q^{2}\right)-\frac{1}{2 \beta_{0}} \frac{\Lambda^{2}}{Q^{2}-\Lambda^{2}}+\ldots,
$$

at the NLO approximation, where the symbol ... stands for terms which have negligible contributions at $Q \geq 1 \mathrm{GeV}[36]^{12}$.

Figure 4 and Table 1 show a strong improvement of the agreement with experimental data for $F_{2}$ (almost 2 times!). Similar results can be seen also in Figs. 5 and 6 for the experimental data for $\lambda_{F_{2}}^{\text {eff }}\left(x, Q^{2}\right)$ at $x \sim 10^{-3}$, which represents an average of the $x$-values of HERA experimental data. The top dashed line represents the aforementioned linear rise of $\lambda\left(Q^{2}\right)$ with $\ln \left(Q^{2}\right)$.

So, Figures 5-7 demonstrate that the theoretical description of the small- $Q^{2}$ ZEUS data for $\lambda_{F_{2}}^{\text {eff }}\left(x, Q^{2}\right)$ by NLO QCD is significantly improved by implementing the "frozen" and analytic coupling constants $\alpha_{\mathrm{fr}}\left(Q^{2}\right)$ and $\alpha_{\mathrm{an}}\left(Q^{2}\right)$, respectively, which in turn lead to very close results (see also $[58,18])$.

\footnotetext{
${ }^{12}$ Note that in $[56,57]$ more accurate, but essentially more cumbersome approximations of $a_{a n}\left(Q^{2}\right)$ have been proposed. We limit ourselves by above simple form (4.2), (4.3) and plan to add the other modifications in our future investigations.
} 


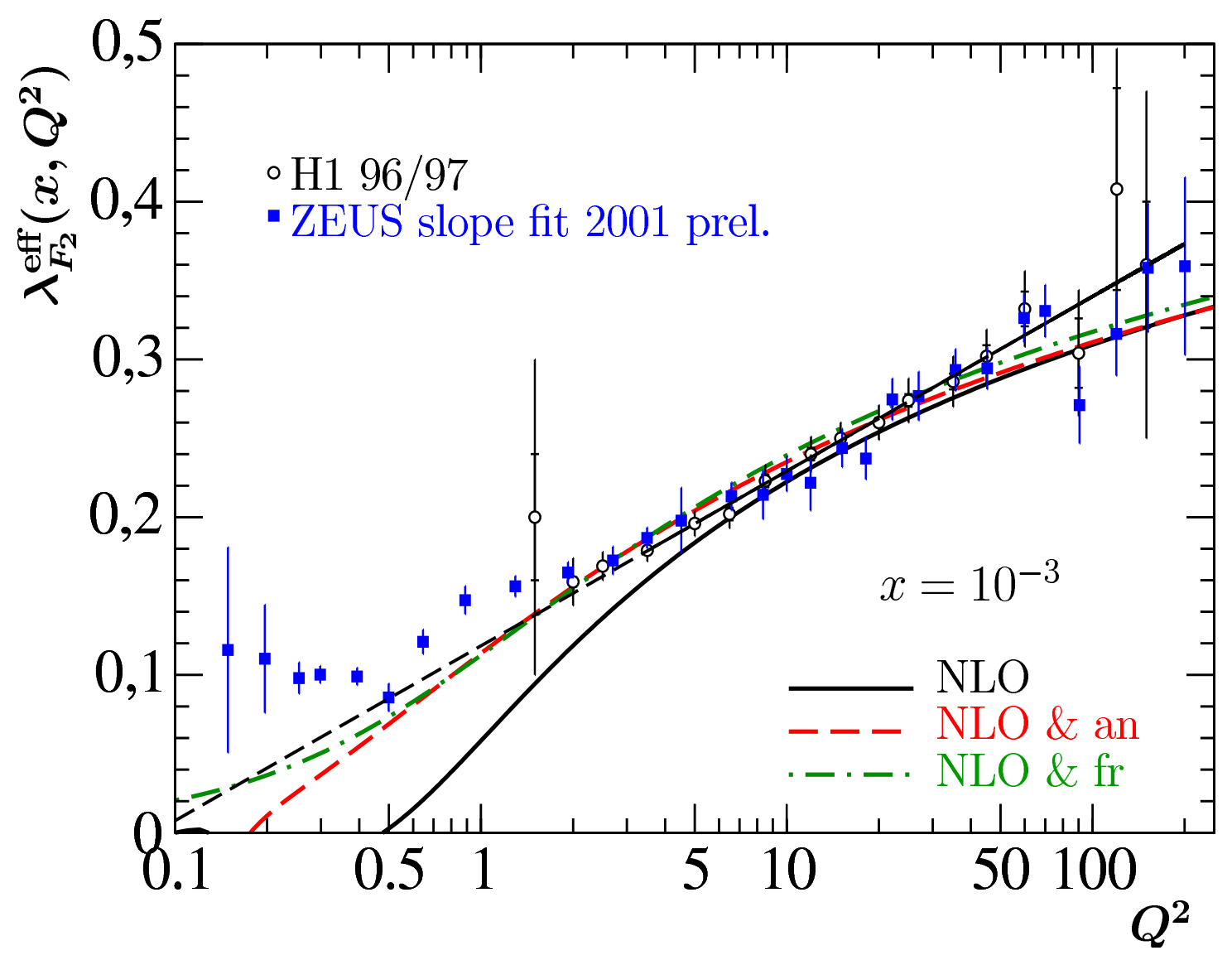

Figure 5: The values of effective slope $\lambda_{\mathrm{F}_{2}}^{\text {eff }}$ as a function of $Q^{2}$ for $x=10^{-3}$. The experimental points are from H1 [5, 6] (open points) and ZEUS [4] (solid points). The solid curve represents the NLO fit. The dash-dotted and lower dashed curves represent the NLO fits with "frozen" and analytic coupling constants, respectively. The top dashed line represents the fit from [5].

Indeed, the fits for $F_{2}\left(x, Q^{2}\right)$ in [22] yielded $Q_{0}^{2} \approx 0.5-0.8 \mathrm{GeV}^{2}$. So, initially we had $\lambda_{F_{2}}^{\text {eff }}\left(x, Q_{0}^{2}\right)=$ 0, as suggested by Eq. (1.1). The replacements of Eqs. (4.1), (4.2) and (4.3) modify the value of $\lambda_{F_{2}}^{\text {eff }}\left(x, Q_{0}^{2}\right)$. For the "frozen" and analytic coupling constants $\alpha_{\mathrm{fr}}\left(Q^{2}\right)$ and $\alpha_{\mathrm{an}}\left(Q^{2}\right)$, the value of $\lambda_{F_{2}}^{\text {eff }}\left(x, Q_{0}^{2}\right)$ is nonzero and the slopes are quite close to the experimental data at $Q^{2} \approx 0.5 \mathrm{GeV}^{2}$. Nevertheless, for $Q^{2} \leq 0.5 \mathrm{GeV}^{2}$, there is still some disagreement with the data, which needs additional investigation. Note that at $Q^{2} \geq 0.5 \mathrm{GeV}^{2}$ our results are even better the results of phenomenological models $[42,66]$.

Figure 7 shows the $x$-dependence of the slope $\lambda_{F_{2}}^{\text {eff }}\left(x, Q^{2}\right)$. One observes good agreement between the experimental data and the generalized DAS approach for a broad range of small- $x$ values. The absence of a variation with $x$ of $\lambda_{F_{2}}^{\text {eff }}\left(x, Q^{2}\right)$ at small $Q^{2}$ values is related to the small values of the variable $\rho$ there.

At large $Q^{2}$ values, the $x$-dependence of $\lambda_{F_{2}}^{\text {eff }}\left(x, Q^{2}\right)$ is rather strong. However, it is well known that the boundaries and mean values of the experimental $x$ ranges [5] increase proportionally with $Q^{2}$, which is related to the kinematical restrictions in the HERA experiments: $x \sim 10^{-4} \times Q^{2}$ (see 


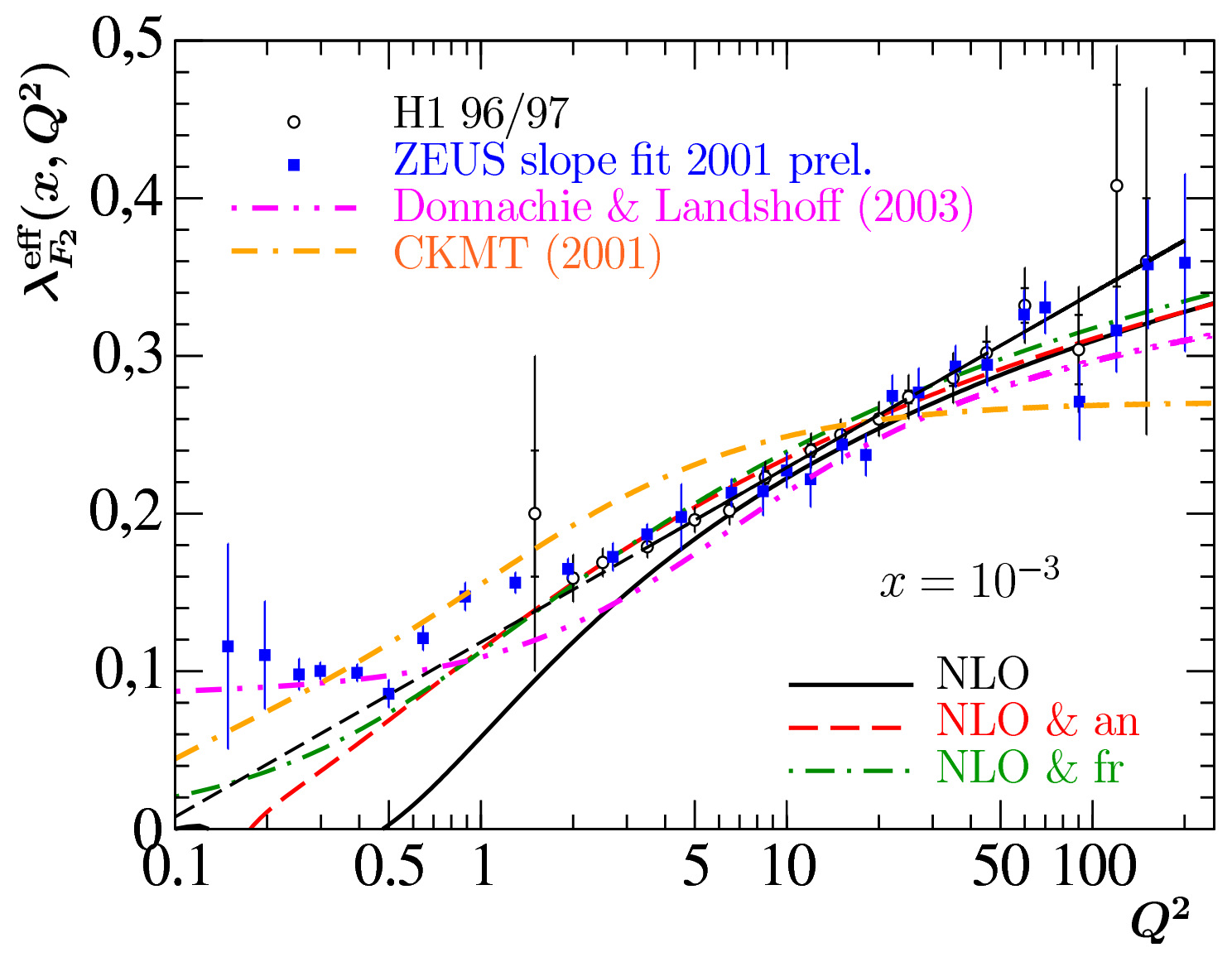

Figure 6: $Q^{2}$ dependence of $\lambda_{\mathrm{F}_{2}}^{\text {eff }}\left(x, Q^{2}\right)$ for an average small- $x$ value of $x=10^{-3}$. The experimental data from H1 (open points) and ZEUS (solid points) are compared with the NLO fits for $Q^{2} \geq 0.5 \mathrm{GeV}^{2}$ implemented with the canonical (solid line), frozen (dot-dashed line), and analytic (dashed line) versions of the strong-coupling constant. The linear rise of $\lambda_{\mathrm{F}_{2}}^{\text {eff }}\left(x, Q^{2}\right)$ with $\ln Q^{2}$ as described by Eq. (2) is indicated by the straight dashed line. For comparison, also the results obtained in the phenomenological models by Capella et al. [42] (dash-dash-dotted line) and by Donnachie and Landshoff [66] (dot-dot-dashed line) are shown.

$[1,2,28]$ and, for example, Fig. 1 of [4]). We show only the case with the "frozen" coupling constant because at large $Q^{2}$ values all results are very similar.

From Fig. 7, one can see that HERA experimental data are close to $\lambda_{F_{2}}^{\text {eff }}\left(x, Q^{2}\right)$ at $x \sim 10^{-4} \div$ $10^{-5}$ for $Q^{2}=4 \mathrm{GeV}^{2}$ and at $x \sim 10^{-2}$ for $Q^{2}=100 \mathrm{GeV}^{2}$. Indeed, the correlations between $x$ and $Q^{2}$ in the form $x_{\text {eff }}=a \times 10^{-4} \times Q^{2}$ with $a=0.1$ and 1 lead to a modification of the $Q^{2}$ evolution which starts to resemble $\ln Q^{2}$, rather than $\ln \ln Q^{2}$ as is standard [28].

\section{Comparison with experimental data for $\mathrm{SF} F_{2}^{c c}$}

We are now in a position to explore the phenomenological implications of our results for SF $F_{2}^{c c}$. As for our input parameters, we choose $m_{c}=1.25 \mathrm{GeV}$ in agreement with Particle Data 


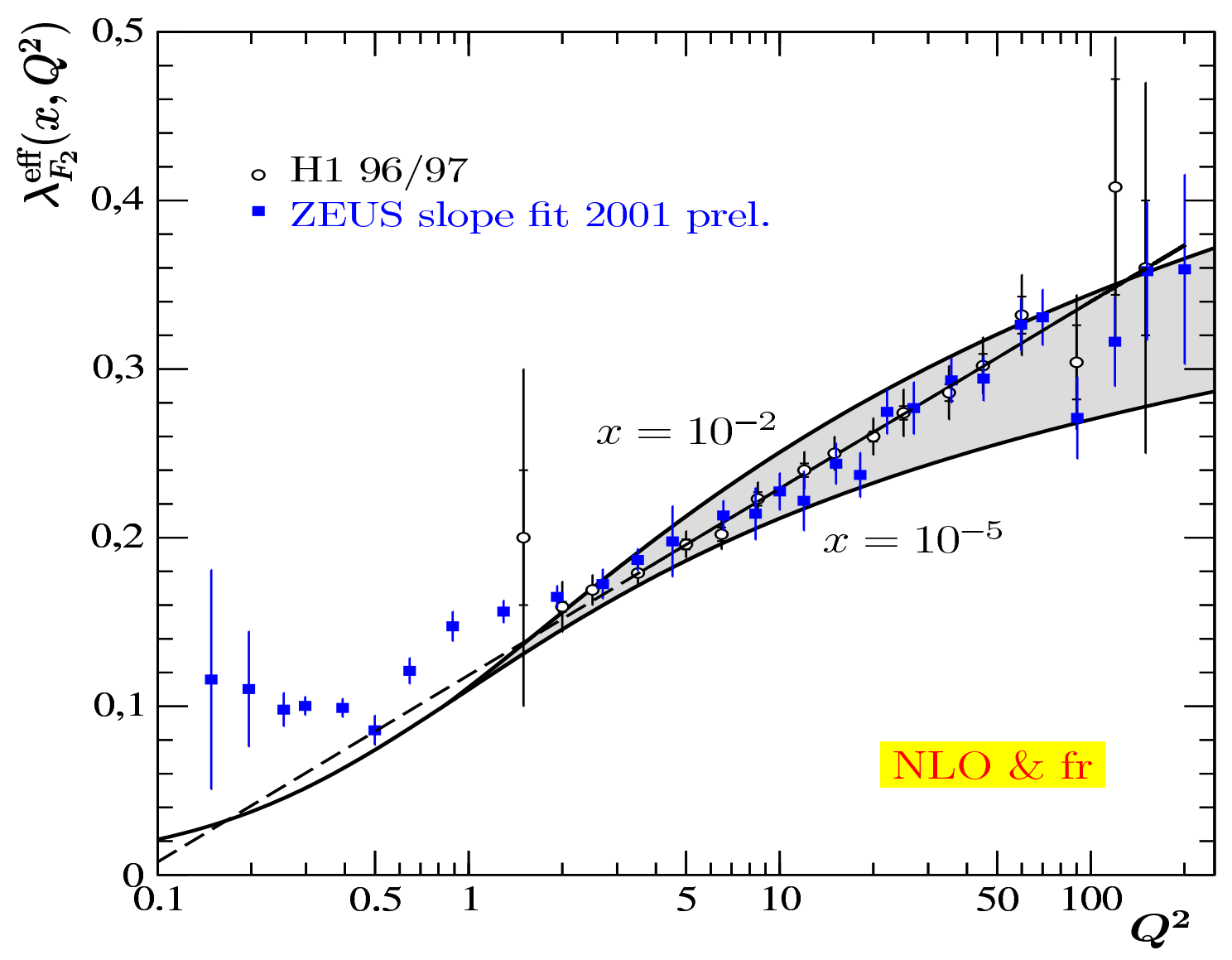

Figure 7: The values of effective slope $\lambda_{\mathrm{F}_{2}}^{\text {eff }}$ as a function of $Q^{2}$. The experimental points are same as on Fig. 4. The dashed line represents the fit from [5]. The solid curves represent the NLO fits with "frozen" coupling constant at $x=10^{-2}$ and $x=10^{-5}$.

Group [67]. While the LO result Eq. (3.7) is independent of the unphysical mass scale $\mu$, the NLO formula (3.2) does depend on it, due to an incomplete compensation of the $\mu$ dependence of $a_{s}(\mu)$ by the terms proportional to $\ln \left(\mu^{2} / Q^{2}\right)$, the residual $\mu$ dependence being formally beyond NLO. In order to fix the theoretical uncertainty resulting from this, we put $\mu^{2}=Q^{2}+4 m_{c}^{2}$ (see (3.3)), which is the standart scale in heavy quark production.

The PDF parameters $\mu_{0}^{2}, A_{q}$ and $A_{g}$ shown in (1.1), have been fixed in the fits of $F_{2}$ experimental data (see the previous section). Their values depend on conditions chosen in the fits: the order of perturbation theory and the number $f$ of active quarks.

Below $b$-quark threshold, the scheme with $f=4$ has been used [22, 29] in the fits of $F_{2}$ data. Note, that the $F_{2}$ structure function contains $F_{2}^{c c}$ as a part. In the fits, the NLO gluon density and the LO and NLO quark ones contribute to $F_{2}^{c}$, as the part of to $F_{2}$. Then, now in PGF scattering the LO coefficient function (3.4) corresponds in $m \rightarrow 0$ limit to the standart NLO Wilson coefficient (together with the product of the LO anomalous dimension $\gamma_{q g}$ and $\ln \left(m_{c}^{2} / Q^{2}\right)$. It is a general situation, i.e. the coefficient funstion of PGF scattering at some order of perturbation theory corresponds to the standart DIS Wilson coefficient with the one step higher order. The reason is following: the 


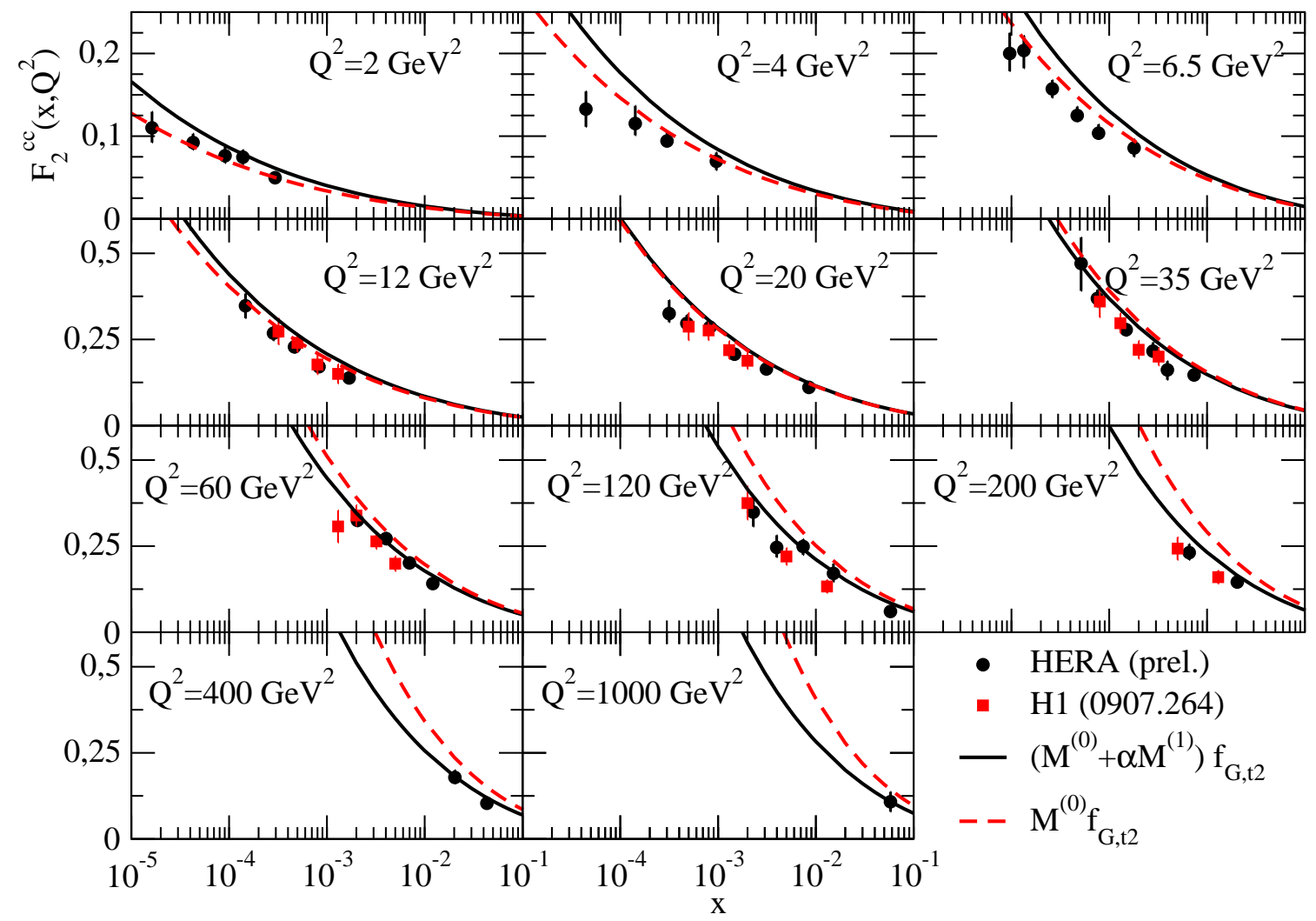

Figure 8: $F_{2}^{c c}\left(x, Q^{2}\right)$ evaluated as functions of $x$ with the LO matrix elements (dashed lines) and with the NLO ones and with the factorization/renormalization scale $\mu^{2}=Q^{2}+4 m_{c}^{2}$ (solid lines). The black points and red squares correspond to the the combine H1ZEUS preliminary data [11] and H1 data [7, 8], respectively.

standart DIS analysis starts with handbag diagram of photon-quark scattering and photon-gluon interaction begins at one-loop level.

Thus, in our $F_{2}^{c c}$ analysis in the LO approximation of PGF process we should take $f_{a}\left(x, Q^{2}\right)$ extracted from fits of $F_{2}$ data at $f=4$ and NLO approximation. In practice, in [49] we have applied our $f=4$ NLO twist-two fit [22] of $\mathrm{H} 1$ data for $F_{2}$ with $Q^{2}$ cut: $Q^{2}>1.5 \mathrm{GeV}^{2}$, which produces $Q_{0}^{2}=0.523 \mathrm{GeV}^{2}, A_{g}=0.060$ and $A_{q}=0.844$.

Correspondingly, the NLO approximation of PGF process needs the gluon density exracted from fits of $F_{2}$ data at NNLO approximation, which is not yet known ${ }^{13}$ in generalized DAS regime. However, we see from the modern global fits [69], that the difference between NLO and NNLO gluon densities is not so large. So, we can apply the NLO form (2.11) of $f_{a}\left(x, Q^{2}\right)$ for our NLO PGF analysis, too.

The results for $F_{2}^{c c}$ are prsented in Fig.8. We can see a good agreement between our compact

\footnotetext{
${ }^{13}$ The difficulty to extend the analysis $[21,22]$ to NNLO level is related with an appearence of the pole $\sim 1 /(n-1)^{2}$ in the three-loop corrections to the anomalous dimension $\gamma_{g g}$ (see [50, 68]). The pole $\sim 1 /(n-1)^{2}$ violates the Bessellike solution (2.11) of DGLAP equation for PDFs at low $x$ values with the flat initial condition (1.1).
} 
formulas (3.1), (3.2), (3.7) and (3.17) an the modern experimental data [7]-[11] for $F_{2}^{c c}\left(x, Q^{2}\right)$ structure function. To keep place on Fig.8, we show only the H1 [7, 8] data and the combine H1ZEUS preliminary [11] one.

The good agreement between generalized double-asymptotic scaling DAS approach used here and $F_{2}$ and $F_{2}^{c c}$ data demonstrates an equal importance of the both parton densities (gluon one and sea quark one) at low $x$. It is due to the fact that $F_{2}$ relates mostly to the sea quark distribution, while the $F_{2}^{c c}$ relates mostly to the gluon one. Dropping sea quarks in analyse ledas to the different gluon densities extracted from $F_{2}$ of from $F_{2}^{c c}$ (see, for example, [70]).

\section{Conclusions}

We have shown the $Q^{2}$-dependence of the structure functions $F_{2}$ and $F_{2}^{c c}$ and of the slope $\lambda_{F_{2}}^{\text {eff }}=\partial \ln F_{2} / \partial \ln (1 / x)$ at small- $x$ values in the framework of perturbative QCD. Our twist-two results are in very good agreement with precise HERA data at $Q^{2} \geq 2 \mathrm{GeV}^{2}$, where perturbative theory can be applicable. The application of the "frozen" and analytic coupling constants $\alpha_{\mathrm{fr}}\left(Q^{2}\right)$ and $\alpha_{\text {an }}\left(Q^{2}\right)$ improves the agreement with the recent HERA data $[4,5,6]$ for the slope $\lambda_{F_{2}}^{\text {eff }}\left(x, Q^{2}\right)$ for small $Q^{2}$ values, $Q^{2} \geq 0.5 \mathrm{GeV}^{2}$.

We presented a compact formula for the heavy-flavour contributions to the proton structure functions $F_{2}$ valid through NLO at small values of Bjorken's $x$ variable. Our results agree with modern experimental data [7]-[11] well within errors without a free additional parameters. In the $Q^{2}$ range probed by the HERA data, our NLO predictions agree very well with the LO ones. Since we worked in the fixed-flavour-number scheme, our results are bound to break down for $Q^{2} \gg 4 m_{i}^{2}$, which manifests itself by appreciable QCD correction factors and scale dependences. As is well known, this problem is conveniently solved by adopting the variable-flavour-number scheme, which not considered here.

As a next step of investigations, we plan to perform combined fits of the H1\&ZEUS data [3] of $F_{2}\left(x, Q^{2}\right)$, the $H 1 \& Z E U S$ data [71] of $F_{2}^{c c}\left(x, Q^{2}\right)$ and the HERA data [7, 9] of $F_{2}^{b b}\left(x, Q^{2}\right)$, using the "frozen" and analytic coupling constants in both the LO and NLO approximations, in order to improve the agreement with HERA data at small $Q^{2}$ values. Several versions of the analytical coupling constant will be used.

\section{Acknowledgments}

This work was supported by RFBR grant 10-02-01259-a. Author thanks the Organizing Committee of XXI International Baldin Seminar on High Energy Physics Problems for invitation and Paolo Bolzoni for discussions.

\section{References}

[1] H1 Collab. (C. Adloff et al.), Nucl. Phys. B 497 (1997) 3; Eur. Phys. J. C 21 (2001) 33.

[2] ZEUS Collab. (S. Chekanov et al.), Eur. Phys. J. C 21 (2001) 443.

[3] H1 and ZEUS Collab. ( F. D. Aaron et al.), JHEP 1001 (2010) 109. 
[4] H1 and ZEUS Collab. (B. Surrow), Phenomenological studies of inclusive e p scattering at low

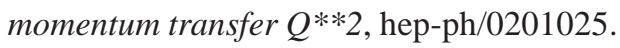

[5] H1 Collab. (C. Adloff et al.), Phys. Lett. B 520 (2001) 183.

[6] H1 Collab. (T. Lastovicka), Acta Phys. Polon. B 33 (2002) 2835; H1 Collab. (J. Gayler), Acta Phys. Polon. B 33 (2002) 2841.

[7] H1 Collab. (F. D. Aaron et al.), Phys. Lett. B 686 (2010) 91.

[8] H1 Collab. (F. D. Aaron et al.), Eur. Phys. J. C 65 (2010) 89.

[9] ZEUS collaboration (H. Abramowicz et al.), Eur. Phys. J. C 69 (2010) 347

[10] ZEUS collaboration (S. Chekanov et al.), Eur. Phys. J. C 65 (2010) 65

[11] H1 and ZEUS Collaboration (K. Lipka), Nucl. Phys. Proc. Suppl. 191 (2009) 163.

[12] V. N. Gribov and L. N. Lipatov, Sov. J. Nucl. Phys. 15 (1972) 438, 675; L. N. Lipatov, Sov. J. Nucl. Phys. 20 (1975) 94; G. Altarelli and G. Parisi, Nucl. Phys. B 126 (1977) 298; Yu. L. Dokshitzer, Sov. Phys. JETP 46 (1977) 641.

[13] A. M. Cooper-Sarkar, R. C. E. Devenish, and A. De Roeck, Int. J. Mod. Phys. A 13 (1998) 3385; A. V. Kotikov, Phys. Part. Nucl. 38 (2007) 1. [Erratum-ibid. 38 (2007) 828].

[14] L. N. Lipatov, Sov. J. Nucl. Phys. 23 (1976) 338; E. A. Kuraev, L. N. Lipatov, and V. S. Fadin, Phys. Lett. B 60 (1975) 50; Sov. Phys. JETP 44 (1976) 443; 45 (1977) 199; Ya. Ya. Balitzki and L. N. Lipatov, Sov. J. Nucl. Phys. 28 (1978) 822; L. N. Lipatov, Sov. Phys. JETP 63 (1986) 904.

[15] A. D. Martin, W. J. Stirling, R. S. Thorne and G. Watt, Eur. Phys. J. C 64 (2009) 653; H. -L. Lai, J. Huston, Z. Li, P. Nadolsky, J. Pumplin, D. Stump and C. -P. Yuan, Phys. Rev. D 82 (2010) 054021; S. Alekhin, J. Blumlein and S. Moch, Phys. Rev. D 86 (2012) 054009; R. D. Ball, V. Bertone, S. Carrazza, C. S. Deans, L. Del Debbio, S. Forte, A. Guffanti and N. P. Hartland et al., Nucl. Phys. B 867 (2013) 244.

[16] P. Jimenez-Delgado and E. Reya, Phys. Rev. D 80 (2009) 114011; Phys. Rev. D 79 (2009) 074023

[17] A. V. Kotikov, G. Parente, and J. Sanchez Guillen, Z. Phys. C 58 (1993) 465; G. Parente, A. V. Kotikov, and V. G. Krivokhizhin, Phys. Lett. B 333 (1994) 190; A. L. Kataev, A. V. Kotikov, G. Parente, and A. V. Sidorov, Phys. Lett. B 388 (1996) 179; Phys. Lett. B 417 (1998) 374; A. L. Kataev, G. Parente, and A. V. Sidorov, Nucl. Phys. B 573 (2000) 405; A. V. Kotikov and V. G. Krivokhijine, Phys. At. Nucl. 68 (2005) 1873; B. G. Shaikhatdenov, A. V. Kotikov, V. G. Krivokhizhin and G. Parente, Phys. Rev. D 81 (2010) 034008 [Erratum-ibid. D 81 (2010) 079904].

[18] A. V. Kotikov, V. G. Krivokhizhin and B. G. Shaikhatdenov, Phys. Atom. Nucl. 75 (2012) 507.

[19] R. D. Ball and S. Forte, Phys. Lett. B 336 (1994) 77.

[20] L. Mankiewicz, A. Saalfeld, and T. Weigl, Phys. Lett. B 393 (1997) 175.

[21] A. V. Kotikov and G. Parente, Nucl. Phys. B 549 (1999) 242; Nucl. Phys. (Proc. Suppl.) A 99 (2001) 196. [hep-ph/0010352].

[22] A. Yu. Illarionov, A. V. Kotikov, and G. Parente, Phys. Part. Nucl. 39 (2008) 307; Nucl. Phys. (Proc. Suppl.) 146 (2005) 234.

[23] A. De Rújula, S. L. Glashow, H. D. Politzer, S.B. Treiman, F. Wilczek, and A. Zee, Phys. Rev. D 10, 1649 (1974) 1649. 
[24] NM Collab. (M. Arneodo et al.), Phys. Lett. B 364 (1995) 107; Nucl. Phys. B 483 (1997) 3; E665 Collab. (M. R. Adams et al.), Phys. Rev. D 54 (1996) 3006; A. Donnachie and P. V. Landshoff, Nucl. Phys. B 244 (1984) 322; 267 (1986) 690; Z. Phys. C 61 (1994) 139.

[25] ZEUS Collab. (J. Breitweg et al.), Phys. Lett. B 407 (1997) 432.

[26] ZEUS Collab. (J. Breitweg et al.), Phys. Lett. B 487 (2000) 53; Eur. Phys. J. C 21 (2001) 443.

[27] F. Schrempp, nstanton-induced processes: An Overview, hep-ph/0507160.

[28] A. V. Kotikov and G. Parente, J. Exp. Theor. Phys. 97 (2003) 859.

[29] G. Cvetic, A. Y. .Illarionov, B. A. Kniehl and A. V. Kotikov, Phys. Lett. B 679 (2009) 350.

[30] A. Y. Illarionov, B. A. Kniehl and A. V. Kotikov, Phys. Lett. B 663 (2008) 66.

[31] S. Albino, P. Bolzoni, B. A. Kniehl and A. Kotikov, Nucl. Phys. B 851 (2011) 86; Nucl. Phys. B 855 (2012) 801 .

[32] P. Bolzoni, B. A. Kniehl and A. V. Kotikov, Gluon and quark jet multiplicities at $N^{3} L O+N N L L$, arXiv:1209.5914 [hep-ph].

[33] P. Bolzoni, Timelike structure functions and hadron multiplicities, arXiv:1211.5550 [hep-ph].

[34] D. I. Kazakov and A. V. Kotikov, Nucl. Phys. B 307 (1988) 721 [Erratum-ibid. B 345 (1990) 299]; Phys. Lett. B 291 (1992) 171; A. V. Kotikov, Phys. Atom. Nucl. 57 (1994) 133 [Yad. Fiz. 57 (1994) 142]; A. V. Kotikov and V. N. Velizhanin, Analytic continuation of the Mellin moments of deep inelastic structure functions, hep-ph/0501274.

[35] G. Curci, M. Greco, and Y. Srivastava, Phys. Rev. Lett. 43 (1979) 834; Nucl. Phys. B 159 (1979) 451; M. Greco, G. Penso, and Y. Srivastava, Phys. Rev. D 21 (1980) 2520; PLUTO Collab. (C. Berger et al.), Phys. Lett. B 100 (1981) 351; N. N. Nikolaev and B. M. Zakharov, Z. Phys. C 49 (1991) 607; 53 (1992) 331; B. Badelek, J. Kwiecinski, and A. Stasto, Z. Phys. C 74 (1997) 297.

[36] D. V. Shirkov and I. L. Solovtsov, Phys. Rev. Lett 79 (1997) 1209; Theor. Math. Phys. 120 (1999) 1220.

[37] C. Lopez and F. J. Ynduráin, Nucl. Phys. B 171 (1980) 231; Nucl. Phys. B 183 (1981) 157; C. Lopez, F. Barreiro, and F. J. Ynduráin, Z. Phys. C 72 (1996) 561; K. Adel, F. Barreiro, and F. J. Ynduráin, Nucl. Phys. B 495 (1997) 221.

[38] A. Donnachie and P. V. Landshoff, Phys. Lett. B 296 (1992) 227; 437 (1998) 408.

[39] H. Abramowitz, E. M. Levin, A. Levy, and U. Maor, Phys. Lett. B 269 (1991) 465; A. V. Kotikov, Mod. Phys. Lett. A 11 (1996) 103; Phys. At. Nucl. 59 (1996) 2137.

[40] A. V. Kotikov, Phys. At. Nucl. 56 (1993) 1276; Phys. Rev. D 49 (1994) 5746.

[41] G. M. Frichter, D. W. McKay, and J. P. Ralston, Phys. Rev. Lett. 74 (1995) 1508.

[42] A. Capella, A. B. Kaidalov, C. Merino, and J. Tran Thanh Van, Phys. Lett. B 337 (1994) 358; A. B. Kaidalov, C. Merino, and D. Pertermann, Eur. Phys. J. C 20 (2001) 301.

[43] P. Desgrolard, L. L. Jenkovszky, and F. Paccanoni, Eur. Phys. J. C 7 (1999) 655; V. I. Vovk, A. V. Kotikov, and S. I. Maximov, Theor. Math. Phys. 84 (1990) 744; L. L. Jenkovszky, A. V. Kotikov, and F. Paccanoni, Sov. J. Nucl. Phys. 55 (1992) 1224; JETP Lett. 58 (1993) 163; Phys. Lett. B 314 (1993) 421; A. V. Kotikov, S. I. Maximov, and I. S. Parobij, Theor. Math. Phys. 111 (1997) 442.

[44] A. D. Martin, W. S. Stirling, and R. G. Roberts, Phys. Lett. B B387 (1996) 419. 
[45] M. Gluck, E. Reya, and A. Vogt, Eur. Phys. J. C 5 (1998) 461; M. Gluck, C. Pisano, and E. Reya, Eur. Phys. J. C 40 (2005) 515.

[46] B. A. Kniehl, G. Kramer and M. Spira, Z. Phys. C 76 (1997) 689; J. Binnewies, B. A. Kniehl and G. Kramer, Z. Phys. C 76 (1997) 677; Phys. Rev. D 58 (1998) 014014; Phys. Rev. D 58 (1998) 034016

[47] S. Frixione, M. L. Mangano, P. Nason and G. Ridolfi, Phys. Lett. B 348 (1995) 633; S. Frixione, P. Nason and G. Ridolfi, Nucl. Phys. B 454 (1995) 3.

[48] F. I. Olness and W. K. Tung, Nucl. Phys. B 308 (1988) 813; M. A. G. Aivazis, F. I. Olness and W. K. Tung, Phys. Rev. D 50 (1994) 3085; M. A. G. Aivazis, J. C. Collins, F. I. Olness and W. K. Tung, Phys. Rev. D 50 (1994) 3102; R. S. Thorne and R. G. Roberts, Phys. Rev. D 57 (1998) 6871; Phys. Lett. B 421 (1998) 303; Eur. Phys. J. C 19 (2001) 339; W. K. Tung, S. Kretzer and C. Schmidt, J. Phys. G 28 (2002) 983.

[49] A. Y. .Illarionov and A. V. Kotikov, Phys. Atom. Nucl. 75 (2012) 1234.

[50] V. S. Fadin and L. N. Lipatov, Phys. Lett. B 429 (1998) 127; G. Camici and M. Ciafaloni, Phys. Lett. B430 (1998) 349.

[51] A. V. Kotikov and L. N. Lipatov, Nucl. Phys. B 582 (2000) 19; Nucl. Phys. B 661 (2003) 19.

[52] G. Grunberg, Phys. Rev. D 29 (1984) 2315; Phys. Lett. B 95 (1980) 70.

[53] Yu. L. Dokshitzer and D. V. Shirkov, Z. Phys. C 67 (1995) 449; A. V. Kotikov, JETP Lett. 59 (1994) 1; Phys. Lett. B 338 (1994) 349; W. K. Wong, Phys. Rev. D 54 (1996) 1094.

[54] S. J. Brodsky, V. S. Fadin, V. T. Kim, L.N. Lipatov, G.B. Pivovarov, JETP. Lett. 70 (1999) 155; M. Ciafaloni, D. Colferai, and G. P. Salam, Phys. Rev. D 60 (1999) 114036 ; JHEP 07 (2000) 054; R. S. Thorne, Phys. Lett. B 474 (2000) 372; Phys. Rev. D 60 (1999) 054031; 64 (2001) 074005; G. Altarelli, R. D. Ball, and S. Forte, Nucl. Phys. B 621 (2002) 359.

[55] Bo Andersson et al., Eur. Phys. J. C 25 (2002) 77.

[56] A. V. Nesterenko, Phys. Rev. D 64 (2001) 116009; Int. J. Mod. Phys. A18 (2003) 5475; A. V. Nesterenko and J. Papavassiliou, Phys. Rev. D 71 (2005) 016009; J. Phys. G 32 (2006) 1025; G. Cvetic, C. Valenzuela, and I. Schmidt, Nucl. Phys. Proc. Suppl. 164 (2007) 308; G. Cvetic and C. Valenzuela, J. Phys. G 32 (2006) L27; Phys. Rev. D 74 (2006) 114030; Phys. Rev. D 77 (2008) 074021; A. P. Bakulev, S. V. Mikhailov, and N. G. Stefanis, Phys. Rev. D 72 (2005) 074014; Phys. Rev. D 75 (2007) 056005; R. S. Pasechnik, D. V. Shirkov, and O. V. Teryaev, Phys. Rev. D 78 (2008) 071902; G. Cvetic and A. V. Kotikov, J. Phys. G 39 (2012) 065005.

[57] G. Cvetic and C. Valenzuela, Braz. J. Phys. 38 (2008) 371; A. P. Bakulev, S. V. Mikhailov, Resummation in (F)APT arXiv:0803.3013 [hep-ph]; N. G. Stefanis, Taming Landau singularities in QCD perturbation theory: The Analytic approach, arXiv:0902.4805 [hep-ph].

[58] A. V. Kotikov, A. V. Lipatov, and N. P. Zotov, J. Exp. Theor. Phys. 101 (2005) 811.

[59] V. N. Baier, V. S. Fadin and V. A. Khoze, Sov. Phys. JETP 23 (1966) 104; V. G. Zima, Yad. Fiz. 16 (1972) 1051; V. M. Budnev, I. F. Ginzburg, G. V. Meledin and V. G. Serbo, Phys. Rept. 15 (1974) 181.

[60] E. Witten, Nucl. Phys. B 104 (1976) 445; J. P. Leveille and T. J. Weiler, Nucl. Phys. B 147 (1979) 147; V. A. Novikov, M. A. Shifman, A. I. Vainshtein and V. I. Zakharov, Nucl. Phys. B 136 (1978) 125

[61] A. V. Kotikov, A. V. Lipatov, G. Parente and N. P. Zotov, Eur. Phys. J. C 26 (2002) 51

[62] A. Y. .Illarionov, B. A. Kniehl and A. V. Kotikov, Phys. Rev. Lett. 106 (2011) 231802. 
[63] E. Laenen, S. Riemersma, J. Smith and W. L. van Neerven, Nucl. Phys. B 392 (1993) 162, 229.

[64] S. Catani, M. Ciafaloni and F. Hautmann, Production of heavy flavors at high-energies, Preprint CERN-Th.6398/92, in Proceeding of the Workshop on Physics at HERA (Hamburg, 1991), Vol. 2., p. 690; S. Catani and F. Hautmann, Nucl. Phys. B 427 (1994) 475; S. Riemersma, J. Smith and W. L. van Neerven, Phys. Lett. B 347 (1995) 143.

[65] P. Nason, S. Dawson and R. K. Ellis, Nucl. Phys. B 303 (1988) 607.

[66] A. Donnachie and P. V. Landshoff, Acta Phys. Polon. B 34 (2003) 2989.

[67] Particle Data Group (C. Amsler et al.) Phys. Lett. B 667 (2008) 1.

[68] A. Vogt, S. Moch and J. A. M. Vermaseren, Nucl. Phys. B 691 (2004) 129

[69] M. Dittmar et al., Working Group I: Parton distributions: Summary report for the HERA LHC Workshop Proceedings, arXiv:hep-ph/0511119.

[70] H. Jung, A. V. Kotikov, A. V. Lipatov and N. P. Zotov, Critical tests of unintegrated gluon distributions, arXiv:0706.3793 [hep-ph]; Towards precision determination of unintegrated parton density functions, arXiv:hep-ph/0611093.

[71] H1 and ZEUS Collaborations (H. Abramowicz et al.), Combination and QCD Analysis of Charm Production Cross Section Measurements in Deep-Inelastic ep Scattering at HERA, arXiv:1211.1182 [hep-ex].

[72] H. Kowalski, L. N. Lipatov and D. A. Ross, BFKL Evolution as a Communicator Between Small and Large Energy Scales, arXiv:1205.6713 [hep-ph].

\section{Appendix A}

Here we give a short introduction of possible accounfing for BFKL corrections to our analysis. As it was shown in Ref. [21] for the first two orders of the perturbation theory, it is conveninet to start with Mellin moment reprentation.

\subsection{Mellin moment form}

In the following we resume the steps we have followed to reach the small $x$ approximate solution of DGLAP shown above (see also [21]) ${ }^{14}$ :

- Use the $n$-space exact solution for " \pm "-components

$$
A_{a}^{ \pm} \exp \left[-\int_{a_{s}\left(Q_{0}^{2}\right)}^{a_{s}\left(Q^{2}\right)} d \tilde{a}_{s} \frac{\gamma_{ \pm}\left(n, \tilde{a}_{s}\right)}{\beta\left(\tilde{a}_{s}\right)}\right] \approx A_{a}^{ \pm} e^{-d_{ \pm}(n) s}
$$

with

$$
d_{ \pm}(n)=-\frac{\gamma_{ \pm}^{(0)}(n)}{\beta_{0}}
$$

- Expand the perturbatively calculated parts (of anomalous dimensions and coefficient functions) in the vicinity of the point $n=1$.

\footnotetext{
${ }^{14}$ To work with BFKL formulas in the most symmetric way, in this Appendix we will use the normalization of the anomalous dimensions deviated by the factor " $-1 / 2$ " from the DIS standard notation.
} 
- The singular part of the "+"-component with the form (hereafter $n=1+\omega$ )

$$
A_{a} \omega^{k} e^{-\hat{d}_{L O} / \omega}
$$

leads to modified Bessel functions in the $x$-space in the form

$$
A_{a}\left(\frac{\hat{d} s}{\ln x}\right)^{(k+1) / 2} I_{k+1}(2 \sqrt{\hat{d} s \ln x})
$$

- The regular part $B(n) \exp (-\bar{d}(n) s)$ leads to the additional coefficient (see Ref. [21] and Appendix there)

$$
B(1) e^{-\bar{d}(1) s}+O(\sqrt{\hat{d} s / \ln x})
$$

behind of the modified Bessel function (A4) in the $x$-space. Because the accuracy is $O\left(\sqrt{\hat{d}_{+} s / \ln x}\right)$, it is necessary to use only the basic term of Eq. (A4), i.e. all terms $\omega^{k}$ in front of $\exp \left(-\hat{d}_{+} / \omega\right)$, with the exception of one with the smaller $k$ value, can be neglected.

- If the singular part at $n \rightarrow 1$ is absent (as in the case of the "-"-component), i.e. $\hat{d}_{-}=0$ in (A3), the result in the $x$-space is determined by $B(1) \exp (-\bar{d}(1) s)$ with accuracy $O(x)$.

\subsection{BFKL corrections}

We would like to stress that the applicability of the above recipe (to constract the small- $x$ solution which was shown in the previous subsection) is not limited by the order in perturbation theory but by the form of the singular part of the anomalous dimensions. At the first two orders of perturbation theory the singular part is proportional to $\sim \omega^{-1}$ but this behaviour does not remain at higher orders. The most singular terms have been calculated in [14]. For example, the singular part of the " + "-component of the anomalous dimension matrix has the following form

$$
\gamma_{+}\left(\omega, a_{s}\right)=\gamma\left(\omega, a_{s}\right)+O\left(a_{s}\left(\frac{a_{s}}{\omega}\right)^{k}\right)
$$

where the terms $\sim O\left(a_{s}\left(a_{s} / \omega\right)^{k}\right)$ have been evaluated in Ref. [50].

The BFKL anomalous dimension $\gamma(\omega, \alpha)$ is obtained by solving the implicit equation

$$
1=\frac{4 C_{A} a_{s}}{\omega} \chi\left(\gamma\left(\omega, a_{s}\right)\right)
$$

where the characteristic function $\chi(\gamma)$ has the following expression in terms of the Euler $\Psi$ function:

$$
\chi(\gamma)=2 \Psi(1)-\Psi(\gamma)-\Psi(1-\gamma), \quad \Psi(\gamma)=\frac{d(\ln (\Gamma(\gamma))}{d \gamma}
$$




\subsubsection{Expansions}

The expansion of $\chi(n, \gamma)$ in powers of $\gamma$ gives:

$$
\chi(\gamma)=\frac{1}{\gamma}+2 \sum_{k=1}^{\infty} \zeta(2 k+3) \gamma^{2 k+2}
$$

that can be rewritten as the following exact relation for $\gamma$ itself

$$
\gamma=\gamma_{0}\left[1+2 \sum_{k=1}^{\infty} \zeta(2 k+3) \gamma^{2 k+3}\right], \quad \gamma_{0}=\frac{4 C_{A} a_{s}}{\omega}
$$

where $\gamma_{0}$ is the singular part of the LO gluon-gluon anomalous dimesion. Solwing above eqution by interations, we have

$$
\gamma=\gamma_{0}+\sum_{k=3}^{\infty} C_{k} \gamma_{0}^{k+1}
$$

where the new coefficients are

$$
C_{3}=2 \zeta(3), C_{4}=0, C_{5}=2 \zeta(5), C_{6}=12 \zeta^{2}(3), C_{7}=2 \zeta(7), \ldots
$$

Incorporating the BFKL term to the renormalization exponent leads to the following replacement

$$
\exp \left\{-\frac{1}{\beta_{0}} \int_{a_{s}\left(Q_{0}^{2}\right)}^{a_{s}\left(Q^{2}\right)} \frac{d a}{a^{2}} \frac{\hat{\gamma}_{+}(a)}{\omega}\right\} \rightarrow \exp \left\{-\frac{1}{\beta_{0}} \int_{a_{s}\left(Q_{0}^{2}\right)}^{a_{s}\left(Q^{2}\right)} \frac{d a}{a^{2}} \gamma(a)\right\}
$$
form

The contribution of the additional term $\sum_{k=3}^{\infty} C_{k} \gamma_{0}^{k+1}$ in the r.h.s. of (A8) has the following

$$
-\frac{1}{\beta_{0}} \int \frac{d a}{a^{2}} \sum_{k=3}^{\infty} C_{k} \gamma_{0}^{k+1}(a)=-\frac{1}{\beta_{0}} \frac{1}{a} \sum_{k=3}^{\infty} \frac{C_{k}}{k} \gamma_{0}^{k+1}(a)
$$

and, thus, it has additional factor $k$ in the denominator. So, it gives a hopeness that in the form (A9) the BFKL contributions will be not so large as usual.

\subsubsection{Exact contribution}

Now we considere the BFKL contribution to the r.h.s. of (A9) without any axpansions. Using integration by parts procedure, we obtain the following expression

$$
\int \frac{d a}{a^{2}} \gamma(a)=-\frac{1}{a} \gamma(a)+\int \frac{d a}{a} \frac{d \gamma(a)}{d a}=-\frac{1}{a} \gamma(a)+\int \frac{d \gamma}{a},
$$

where the inverse coupling constant is proportioanl to the characteristic function $\chi(\gamma)$ in (A6)

$$
\frac{1}{a}=\frac{4 C_{A}}{\omega} \chi(\gamma)
$$


Thus, the last integral in (A10) can be evaluated exactly as

$$
\int d \gamma \chi(\gamma)=2 \Psi(1) \gamma+\ln \frac{\Gamma(1-\gamma)}{\Gamma(\gamma)}
$$

So, the needed contribution in the r.h.s. of (A9) can be represented in the following form

$$
\exp \left\{-\frac{1}{\beta_{0}} \int_{a_{s}\left(Q_{0}^{2}\right)}^{a_{s}\left(Q^{2}\right)} \frac{d a}{a^{2}} \gamma(a)\right\}=\frac{R\left(Q^{2}\right)}{R\left(Q_{0}^{2}\right)},
$$

where the $R$ value is (see also Ref. [72])

$$
R\left(Q^{2}\right)=\left[\frac{\Gamma(\gamma)}{\Gamma(1-\gamma)}\right]^{d_{0}} \exp \left\{-d_{0} \gamma(\Psi(\gamma)+\Psi(1-\gamma))\right\}
$$

with the new parameter $d_{0}$

$$
d_{0}=\frac{4 C_{A}}{\beta_{0} \omega}=-\frac{\hat{d}_{+}}{\omega}=-\frac{\hat{d}_{g g}}{\omega}
$$

When $a_{s} \rightarrow 0$ (i.e. in the considered case $\gamma \rightarrow 0$ ) we recover the singular part of the LO contributions (see the previous section)

$$
R\left(Q^{2}\right) \rightarrow \gamma^{-d_{0}} \rightarrow a_{s}^{-d_{0}}
$$

The transform of the Mellin moments in the form (A11) and (A12) to the Bjorken $x$-space, it is not a trivial problem. Author plans to return to this problem in his future work. 